EUROPEAN ORGANIZATION FOR NUCLEAR RESEARCH

CERN-PPE/95-127

22 August 1995

\title{
Tau leptonic branching ratios
}

\author{
The ALEPH Collaboration*
}

\begin{abstract}
A sample of $62249 \tau$-pair events is selected from data taken with the ALEPH detector in 1991, 1992 and 1993. The measurement of the branching fractions for $\tau$ decays into electrons and muons is presented with emphasis on the study of systematic effects from selection, particle identification and decay classification. The results obtained are: $B_{e}=17.79 \pm 0.12($ stat $) \pm 0.06($ syst $)(\%)$ and $B_{\mu}=17.31 \pm 0.11($ stat $) \pm 0.05($ syst $)(\%)$. Combined with the most recent ALEPH determination of the $\tau$ lifetime, these results provide a relative measurement of the leptonic couplings in the weak charged current for transverse $\mathrm{W}$ bosons: $g_{\mu} / g_{e}=1.0002 \pm 0.0051$ and $g_{\tau} / g_{\mu}=0.9943 \pm 0.0065$.
\end{abstract}

(Submitted to Zeitschrift für Physik $\mathbf{C}$ )

*) See next pages for the list of authors 


\section{The ALEPH Collaboration}

D. Buskulic, D. Casper, I. De Bonis, D. Decamp, P. Ghez, C. Goy, J.-P. Lees, A. Lucotte, M.-N. Minard, P. Odier, B. Pietrzyk

Laboratoire de Physique des Particules (LAPP), IN ${ }^{2} P^{3}$-CNRS, 74019 Annecy-le-Vieux Cedex, France

F. Ariztizabal, M. Chmeissani, J.M. Crespo, I. Efthymiopoulos, E. Fernandez, M. Fernandez-Bosman, V. Gaitan, Ll. Garrido, ${ }^{15}$ M. Martinez, S. Orteu, A. Pacheco, C. Padilla, F. Palla, A. Pascual, J.A. Perlas, F. Sanchez, F. Teubert

Institut de Fisica d'Altes Energies, Universitat Autonoma de Barcelona, 08193 Bellaterra (Barcelona), Spain $^{7}$

A. Colaleo, D. Creanza, M. de Palma, A. Farilla, G. Gelao, M. Girone, G. Iaselli, G. Maggi, ${ }^{3}$ M. Maggi, N. Marinelli, S. Natali, S. Nuzzo, A. Ranieri, G. Raso, F. Romano, F. Ruggieri, G. Selvaggi, L. Silvestris, P. Tempesta, G. Zito

Dipartimento di Fisica, INFN Sezione di Bari, 70126 Bari, Italy

X. Huang, J. Lin, Q. Ouyang, T. Wang, Y. Xie, R. Xu, S. Xue, J. Zhang, L. Zhang, W. Zhao

Institute of High-Energy Physics, Academia Sinica, Beijing, The People's Republic of China ${ }^{8}$

G. Bonvicini, M. Cattaneo, P. Comas, P. Coyle, H. Drevermann, A. Engelhardt, R.W. Forty, M. Frank, R. Hagelberg, J. Harvey, R. Jacobsen, ${ }^{24}$ P. Janot, B. Jost, E. Kneringer, J. Knobloch, I. Lehraus, C. Markou, ${ }^{23}$

E.B. Martin, P. Mato, A. Minten, R. Miquel, T. Oest, P. Palazzi, J.R. Pater, ${ }^{27}$ J.-F. Pusztaszeri, F. Ranjard, P. Rensing, L. Rolandi, D. Schlatter, M. Schmelling, O. Schneider, W. Tejessy, I.R. Tomalin, A. Venturi, H. Wachsmuth, W. Wiedenmann, T. Wildish, W. Witzeling, J. Wotschack

European Laboratory for Particle Physics (CERN), 1211 Geneva 23, Switzerland

Z. Ajaltouni, M. Bardadin-Otwinowska, ${ }^{2}$ A. Barres, C. Boyer, A. Falvard, P. Gay, C. Guicheney, P. Henrard, J. Jousset, B. Michel, S. Monteil, J-C. Montret, D. Pallin, P. Perret, F. Podlyski, J. Proriol, J.-M. Rossignol, F. Saadi

Laboratoire de Physique Corpusculaire, Université Blaise Pascal, IN ${ }^{2} P^{3}$-CNRS, Clermont-Ferrand, 63177 Aubière, France

T. Fearnley, J.B. Hansen, J.D. Hansen, J.R. Hansen, P.H. Hansen, B.S. Nilsson

Niels Bohr Institute, 2100 Copenhagen, Denmark ${ }^{9}$

A. Kyriakis, E. Simopoulou, I. Siotis, A. Vayaki, K. Zachariadou

Nuclear Research Center Demokritos (NRCD), Athens, Greece

A. Blondel, ${ }^{21}$ G. Bonneaud, J.C. Brient, P. Bourdon, L. Passalacqua, A. Rougé, M. Rumpf, R. Tanaka, A. Valassi, ${ }^{6}$ M. Verderi, H. Videau

Laboratoire de Physique Nucléaire et des Hautes Energies, Ecole Polytechnique, IN ${ }^{2} P^{3}-C N R S, 91128$

Palaiseau Cedex, France

D.J. Candlin, M.I. Parsons

Department of Physics, University of Edinburgh, Edinburgh EH9 3JZ, United Kingdom ${ }^{10}$

E. Focardi, G. Parrini

Dipartimento di Fisica, Università di Firenze, INFN Sezione di Firenze, 50125 Firenze, Italy

M. Corden, M. Delfino, ${ }^{12}$ C. Georgiopoulos, D.E. Jaffe 
Supercomputer Computations Research Institute, Florida State University, Tallahassee, FL 32306$4052, \operatorname{USA}^{13,14}$

A. Antonelli, G. Bencivenni, G. Bologna, ${ }^{4}$ F. Bossi, P. Campana, G. Capon, V. Chiarella, G. Felici, P. Laurelli, G. Mannocchi, ${ }^{5}$ F. Murtas, G.P. Murtas, M. Pepe-Altarelli

Laboratori Nazionali dell'INFN (LNF-INFN), 00044 Frascati, Italy

S.J. Dorris, A.W. Halley, I. ten Have, ${ }^{6}$ I.G. Knowles, J.G. Lynch, W.T. Morton, V. O'Shea, C. Raine, P. Reeves, J.M. Scarr, K. Smith, M.G. Smith, A.S. Thompson, F. Thomson, S. Thorn, R.M. Turnbull

Department of Physics and Astronomy, University of Glasgow, Glasgow G12 8QQ,United Kingdom ${ }^{10}$

U. Becker, O. Braun, C. Geweniger, G. Graefe, P. Hanke, V. Hepp, E.E. Kluge, A. Putzer, B. Rensch,

M. Schmidt, J. Sommer, H. Stenzel, K. Tittel, S. Werner, M. Wunsch

Institut für Hochenergiephysik, Universität Heidelberg, 69120 Heidelberg, Fed. Rep. of Germany ${ }^{16}$

R. Beuselinck, D.M. Binnie, W. Cameron, D.J. Colling, P.J. Dornan, N. Konstantinidis, L. Moneta,

A. Moutoussi, J. Nash, G. San Martin, J.K. Sedgbeer, A.M. Stacey

Department of Physics, Imperial College, London SW7 2BZ, United Kingdom ${ }^{10}$

G. Dissertori, P. Girtler, D. Kuhn, G. Rudolph

Institut für Experimentalphysik, Universität Innsbruck, 6020 Innsbruck, Austria $^{18}$

C.K. Bowdery, T.J. Brodbeck, P. Colrain, G. Crawford, A.J. Finch, F. Foster, G. Hughes, T. Sloan, E.P. Whelan, M.I. Williams

Department of Physics, University of Lancaster, Lancaster LA1 4YB, United Kingdom ${ }^{10}$

A. Galla, A.M. Greene, K. Kleinknecht, G. Quast, J. Raab, B. Renk, H.-G. Sander, R. Wanke, P. van Gemmeren C. Zeitnitz

Institut für Physik, Universität Mainz, 55099 Mainz, Fed. Rep. of Germany ${ }^{16}$

J.J. Aubert, A.M. Bencheikh, C. Benchouk, A. Bonissent, ${ }^{21}$ G. Bujosa, D. Calvet, J. Carr, C. Diaconu,

F. Etienne, M. Thulasidas, D. Nicod, P. Payre, D. Rousseau, M. Talby

Centre de Physique des Particules, Faculté des Sciences de Luminy, IN ${ }^{2} P^{3}-C N R S, 13288$ Marseille, France

I. Abt, R. Assmann, C. Bauer, W. Blum, D. Brown, ${ }^{24}$ H. Dietl, F. Dydak,${ }^{21}$ G. Ganis, C. Gotzhein, K. Jakobs, H. Kroha, G. Lütjens, G. Lutz, W. Männer, H.-G. Moser, R. Richter, A. Rosado-Schlosser, S. Schael,

R. Settles, H. Seywerd, R. St. Denis, G. Wolf

Max-Planck-Institut für Physik, Werner-Heisenberg-Institut, 80805 München, Fed. Rep. of Germany ${ }^{16}$

R. Alemany, J. Boucrot, O. Callot, A. Cordier, F. Courault, M. Davier, L. Duflot, J.-F. Grivaz, Ph. Heusse,

M. Jacquet, D.W. Kim ${ }^{19}$ F. Le Diberder, J. Lefrançois, A.-M. Lutz, G. Musolino, I. Nikolic, H.J. Park,

I.C. Park, M.-H. Schune, S. Simion, J.-J. Veillet, I. Videau

Laboratoire de l'Accélérateur Linéaire, Université de Paris-Sud, IN ${ }^{2} P^{3}-C N R S, 91405$ Orsay Cedex, France

D. Abbaneo, P. Azzurri, G. Bagliesi, G. Batignani, S. Bettarini, C. Bozzi, G. Calderini, M. Carpinelli,

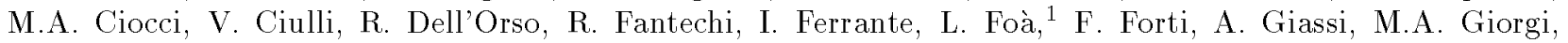

A. Gregorio, F. Ligabue, A. Lusiani, P.S. Marrocchesi, A. Messineo, G. Rizzo, G. Sanguinetti, A. Sciabà,

P. Spagnolo, J. Steinberger, R. Tenchini, G. Tonelli, ${ }^{26}$ G. Triggiani, C. Vannini, P.G. Verdini, J. Walsh

Dipartimento di Fisica dell'Università, INFN Sezione di Pisa, e Scuola Normale Superiore, 56010 Pisa, Italy

A.P. Betteridge, G.A. Blair, L.M. Bryant, F. Cerutti, Y. Gao, M.G. Green, D.L. Johnson, T. Medcalf, Ll.M. Mir, P. Perrodo, J.A. Strong

Department of Physics, Royal Holloway \& Bedford New College, University of London, Surrey TW20 OEX, United Kingdom ${ }^{10}$

V. Bertin, D.R. Botterill, R.W. Clifft, T.R. Edgecock, S. Haywood, M. Edwards, P. Maley, P.R. Norton, J.C. Thompson

Particle Physics Dept., Rutherford Appleton Laboratory, Chilton, Didcot, Oxon OX11 OQX, United Kingdom ${ }^{10}$ 
B. Bloch-Devaux, P. Colas, S. Emery, W. Kozanecki, E. Lançon, M.C. Lemaire, E. Locci, B. Marx, P. Perez, J. Rander, J.-F. Renardy, A. Roussarie, J.-P. Schuller, J. Schwindling, A. Trabelsi, B. Vallage

CEA, DAPNIA/Service de Physique des Particules, CE-Saclay, 91191 Gif-sur-Yvette Cedex, France ${ }^{17}$

R.P. Johnson, H.Y. Kim, A.M. Litke, M.A. McNeil, G. Taylor

Institute for Particle Physics, University of California at Santa Cruz, Santa Cruz, CA 95064, USA ${ }^{22}$

A. Beddall, C.N. Booth, R. Boswell, S. Cartwright, F. Combley, I. Dawson, A. Koksal, M. Letho, W.M. Newton, C. Rankin, L.F. Thompson

Department of Physics, University of Sheffield, Sheffield S3 7RH, United Kingdom ${ }^{10}$

A. Böhrer, S. Brandt, G. Cowan, E. Feigl, C. Grupen, G. Lutters, J. Minguet-Rodriguez, F. Rivera, ${ }^{25}$ P. Saraiva, L. Smolik, F. Stephan,

Fachbereich Physik, Universität Siegen, 57068 Siegen, Fed. Rep. of Germany ${ }^{16}$

M. Apollonio, L. Bosisio, R. Della Marina, G. Giannini, B. Gobbo, F. Ragusa ${ }^{20}$

Dipartimento di Fisica, Università di Trieste e INFN Sezione di Trieste, 34127 Trieste, Italy

J. Rothberg, S. Wasserbaech

Experimental Elementary Particle Physics, University of Washington, WA 98195 Seattle, U.S.A.

S.R. Armstrong, L. Bellantoni, ${ }^{30}$ P. Elmer, Z. Feng, D.P.S. Ferguson, Y.S. Gao, S. González, J. Grahl, J.L. Harton, ${ }^{28}$ O.J. Hayes, H. Hu, P.A. McNamara III, J.M. Nachtman, W. Orejudos, Y.B. Pan, Y. Saadi, M. Schmitt, I.J. Scott, V. Sharma, ${ }^{29}$ J.D. Turk, A.M. Walsh, Sau Lan Wu, X. Wu, J.M. Yamartino, M. Zheng, G. Zobernig

Department of Physics, University of Wisconsin, Madison, WI 53706, USA ${ }^{11}$

\footnotetext{
${ }^{1}$ Now at CERN, 1211 Geneva 23, Switzerland.

${ }^{2}$ Deceased.

${ }^{3}$ Now at Dipartimento di Fisica, Università di Lecce, 73100 Lecce, Italy.

${ }^{4}$ Also Istituto di Fisica Generale, Università di Torino, Torino, Italy.

${ }^{5}$ Also Istituto di Cosmo-Geofisica del C.N.R., Torino, Italy.

${ }^{6}$ Supported by the Commission of the European Communities, contract ERBCHBICT 941234.

${ }^{7}$ Supported by CICYT, Spain.

${ }^{8}$ Supported by the National Science Foundation of China.

${ }^{9}$ Supported by the Danish Natural Science Research Council.

${ }^{10}$ Supported by the UK Particle Physics and Astronomy Research Council.

${ }^{11}$ Supported by the US Department of Energy, grant DE-FG0295-ER40896.

${ }^{12}$ On leave from Universitat Autonoma de Barcelona, Barcelona, Spain.

${ }^{13}$ Supported by the US Department of Energy, contract DE-FG05-92ER40742.

${ }^{14}$ Supported by the US Department of Energy, contract DE-FC05-85ER250000.

${ }^{15}$ Permanent address: Universitat de Barcelona, 08208 Barcelona, Spain.

${ }^{16}$ Supported by the Bundesministerium für Forschung und Technologie, Fed. Rep. of Germany.

${ }^{17}$ Supported by the Direction des Sciences de la Matière, C.E.A.

${ }^{18}$ Supported by Fonds zur Förderung der wissenschaftlichen Forschung, Austria.

${ }^{19}$ Permanent address: Kangnung National University, Kangnung, Korea.

${ }^{20}$ Now at Dipartimento di Fisica, Università di Milano, Milano, Italy.

${ }^{21}$ Also at CERN, 1211 Geneva 23, Switzerland.

${ }^{22}$ Supported by the US Department of Energy, grant DE-FG03-92ER40689.

${ }^{23}$ Now at University of Athens, 157-71 Athens, Greece.

${ }^{24}$ Now at Lawrence Berkeley Laboratory, Berkeley, CA 94720, USA.

${ }^{25}$ Partially supported by Colciencias, Colombia.

${ }^{26}$ Also at Istituto di Matematica e Fisica, Università di Sassari, Sassari, Italy.

${ }^{27}$ Now at Schuster Laboratory, University of Manchester, Manchester M13 9PL, UK.

${ }^{28}$ Now at Colorado State University, Fort Collins, CO 80523, USA.

${ }^{29}$ Now at University of California at San Diego, La Jolla, CA 92093, USA.

${ }^{30}$ Now at Fermi National Accelerator Laboratory, Batavia, IL 60510, USA.
} 


\section{Contents}

1 Introduction $\quad 1$

2 The ALEPH detector 2

3 Particle identification $\quad 3$

3.1 Likelihood identification method . . . . . . . . . . . . 3

3.2 Selection of tracks and discriminating variables . . . . . . . . . . . . 4

3.3 Procedure and results . . . . . . . . . . . . . . . . 5

3.4 Measurement of identification efficiencies . . . . . . . . . . . . . 5

4 The selection of $\tau \tau$ events $\quad 10$

4.1 Preselection procedure ................................. 10

4.2 Further selection using the likelihood particle identification . . . . . . . . 11

4.3 Estimate of remaining backgrounds . . . . . . . . . . . . . . 14

4.4 Measurement of selection efficiencies ................. 18

5 Final definition of the leptonic decays $\quad 21$

5.1 Requirement of a single track . . . . . . . . . . . . . . . 21

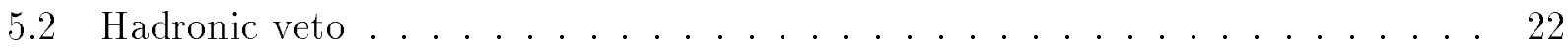

6 Additional systematic studies $\quad 22$

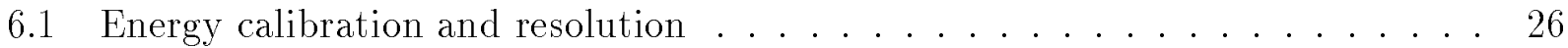

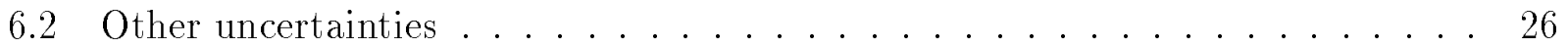

$\begin{array}{llr}7 & \text { Results } & 27\end{array}$

8 Tests of lepton universality in W couplings $\quad 32$

8.1 Test of $\mu-e$ universality . . . . . . . . . . . . . . . . . . . . . . . . . . . . . .

8.2 Tests of $\tau-\mu$ and $\tau$-e universality . . . . . . . . . . . . . 34

$\begin{array}{llr}9 & \text { Conclusions } & 37\end{array}$ 


\section{Introduction}

Universality of couplings between different quark-lepton families is a basic assumption of the Standard Model. In the lepton sector, this hypothesis can be investigated in both the neutral and charged weak currents. Whereas universality is observed to hold within 0.003 for the neutral couplings [1], the situation is less advanced for the charged couplings because W decays so far have not been studied at the same level of precision already achieved in $\mathrm{Z}$ decays. A more practical way to explore the charged couplings is the comparison of lepton decay rates, i.e., the measurement of the purely leptonic decays $\mu^{-} \rightarrow \nu_{\mu} e^{-} \bar{\nu}_{e}, \tau^{-} \rightarrow \nu_{\tau} e^{-} \bar{\nu}_{e}$ and $\tau^{-} \rightarrow \nu_{\tau} \mu^{-} \bar{\nu}_{\mu}$.

The leptonic widths can be computed in the Standard Model including radiative corrections with essentially no uncertainties [2]. On the experimental side, the determination of the $\tau$ leptonic widths involves the measurements of the $\tau$ lifetime and of the electron $\left(B_{e}\right)$ and muon $\left(B_{\mu}\right)$ branching fractions. The universality test also requires the knowledge of the $\tau$ mass and the mass and lifetime of the muon which are known with high precision [3]. The experimental situation in this field has been somewhat unclear in the past with some discrepancy observed with respect to universality [4].

Further motivation to measure $B_{e}$ and $B_{\mu}$ with high precision is provided by the fact that the hadronic branching fraction, i.e., $\left(1-B_{e}-B_{\mu}\right)$, is sensitive to QCD corrections, hence allowing a precise measurement of the strong coupling constant at the $\tau$ mass scale $[5,6,7]$.

In this paper, new precise results on $B_{e}$ and $B_{\mu}$ are reported from samples of 20571 electronic and 20745 muonic $\tau$ decays obtained in $\tau$ pairs produced in $e^{+} e^{-}$collisions at LEP near the Z peak energy in 1991, 1992 and 1993. The fraction of the data taken at the peak energy is $84.5 \%$. Particular care is given to the study and the reduction of systematic effects which could affect the processes of selection, particle identification and decay channel classification.

The analysis starts with a selection of $\tau \tau$ events $(N \tau \tau \sim 62000)$ with a large overall efficiency $\left(\varepsilon_{\tau \tau}^{s e l} \sim 78 \%\right)$ and a small contamination from background processes $\left(f_{\tau \tau}^{n o n^{-} \tau} \sim 0.9 \%\right)$. The $\tau \tau$ selection has a large efficiency for the leptonic decays $\left(\varepsilon_{l}^{\text {sel }} \sim 77 \%\right.$ for electrons, $\sim 79 \%$ for muons) and results in a small non- $\tau$ background contamination in the lepton samples $\left(f_{l}^{n o n^{-}} \sim 1.2 \%\right.$ for electrons, $\sim 0.9 \%$ for muons). Most of the inefficiency in the selection comes from the geometical acceptance of $\sim 85 \%$. Leptons are identified efficiently $\left(N_{l}\right.$ decays with an identification efficiency $\varepsilon_{l}^{I D} \sim 93 \%$ for electrons, $\sim 94 \%$ for muons) with a small contamination from $\tau$ hadronic decays $\left(f_{h \rightarrow l}^{\tau} \sim 1.3 \%\right.$ for electrons, $\sim 1.1 \%$ for muons $)$. The branching ratios are obtained through the expression

$$
B_{l}=\frac{N_{l}\left(1-f_{l}^{\text {non- } \tau}-f_{h \rightarrow l}^{\tau}\right)}{2 N_{\tau \tau}\left(1-f_{\tau}^{n o \tau^{-} \tau}\right)}\left(\frac{\varepsilon_{\tau \tau}^{\text {sel }}}{\varepsilon_{l}^{s e l}}\right) \frac{1}{\varepsilon_{l}^{I D}},
$$

where $l$ stands for either electron or muon. 
In order to achieve a systematic uncertainty of $10^{-3}$, the preevaluations for backgrounds and efficiencies from the simulation are corrected systematically with detailed comparisons to the data. Throughout the analysis the standard V-A matrix element is assumed as supported by Ref. [8].

\section{The ALEPH detector}

A detailed description of the ALEPH detector is given elsewhere [9]. Charged particles are detected successively by a silicon-strip vertex detector (VDET), a drift-cell inner tracking chamber (ITC) and a large-volume time projection chamber (TPC). Beyond the TPC, the electromagnetic calorimeter (ECAL) identifies photons and electrons while the hadronic calorimeter (HCAL) detects the showers produced by hadrons and separates out the muons which are further measured by two layers of streamer-tube chambers placed around HCAL. The inner volume including ECAL is immersed in a 1.5-T axial magnetic field produced by a superconducting solenoidal coil. The return yoke of the magnet provides the sampling absorber material for HCAL.

The ITC cells have a maximum drift time of $130 \mathrm{~ns}$, and its precise timing helps to reduce the cosmic background. The TPC is optimized to accurately measure the particle threemomenta through a maximum of 21 space points with $r \phi$ (transverse to the beams) and $z$ (along the beams) precisions of $170 \mu \mathrm{m}$ and $740 \mu \mathrm{m}$, respectively. The transverse momentum resolution using VDET, ITC and TPC is

$$
\frac{\sigma\left(p_{T}\right)}{p_{T}}=6 \cdot 10^{-4} p_{T}(\mathrm{GeV} / c) \oplus 0.005
$$

In addition to its principal role as a tracking device, the TPC also measures the ionization loss $(d E / d x)$ providing an independent identification tool. For one-prong $\tau$ decays a very precise $d E / d x$ calibration can be performed [10] since the charged particle is isolated. The separation between electrons and pions is larger than $4 \sigma$ up to $8 \mathrm{GeV} / c$.

Since the photon multiplicity is relatively large in $\tau$ decays it is important to understand their conversion in the detector. The amount of material expressed in radiation lengths seen by a particle emerging perpendicularly to the beams is $0.3 \%$ for the beam pipe, $4.1 \%$ for VDET, $0.3 \%$ (1.0\%) for the ITC inner (outer) wall and $2.3 \%$ for the TPC inner wall. Detailed checks of the Monte Carlo simulation of conversions are made with the data and show good agreement for the description of the detector material.

The fine granularity of ECAL is a key feature of the ALEPH detector for $\tau$ physics. The barrel and end cap parts of ECAL are divided into 12 modules, each covering $30^{\circ}$ azimuthally. The modules are built with 45 layers of lead and proportional wire chambers, and the cathode 
pads are read out along projective towers. Fine granularity is achieved transversally and longitudinally: each tower covers a solid angle of $0.9^{\circ} \times 0.9^{\circ}$ and the 45 layers are regrouped into three stacks of 4,9 , and 9 radiation lengths, respectively. The energy of photons and electrons is measured with a resolution of

$$
\frac{\sigma(E)}{E}=\frac{0.18}{\sqrt{E(\mathrm{GeV})}}+0.009
$$

Like ECAL the hadron calorimeter is composed of a barrel closed by two end caps. Its depth of 7.2 interaction lengths is adequate for absorbing hadrons. The digital pattern provided by the read out of the 23 planes of streamer tubes gives a two-dimensional picture of hadronic showers and allows an easy separation of charged hadrons from penetrating minimum-ionizing muons. Cathode pads are organized with a structure analogous to that of ECAL, however with a larger tower size of $3.7^{\circ} \times 3.7^{\circ}$. The muon chambers provide threedimensional hits which can be associated to penetrating tracks.

Finally the three-level trigger system is based on redundant requirements derived from ITC charged-track elements and energy deposits in ECAL and HCAL, used individually or correlated in space. The trigger efficiency within the detector acceptance and the selection cuts is better than $99.99 \%$ for $\tau \tau$ events.

\section{Particle identification}

Charged particle identification plays a role in the measurement of leptonic branching ratios. In this analysis, a likelihood method is used to incorporate the information from the relevant detectors. In this way, each charged particle is assigned a set of probabilities with which a particle type is chosen.

\subsection{Likelihood identification method}

A set of discriminating variables $x_{i}$ is selected and the corresponding probability densities $f_{i}^{j}\left(x_{i}\right)$ for given particle types $j$ are set up using the ALEPH Monte Carlo simulation. Each charged particle is assigned to the type with the largest global estimator $P_{j}$ defined as

$$
P_{j}=\frac{\prod_{i} f_{i}^{j}\left(x_{i}\right)}{\sum_{j} \prod_{i} f_{i}^{j}\left(x_{i}\right)},
$$

where $j=e, \mu, h$. No attempt is made at this point to separate pions from kaons in the hadron sample. 
A first version of this likelihood method was used in a previous analysis [11]. In the present work the following improvements are made: (i) cracks between ECAL modules are defined geometrically on the basis of the track extrapolation, (ii) muon chamber information is now used, (iii) an additional variable from HCAL is introduced for $\pi-\mu$ separation, (iv) reference distributions for each variable are set up separately in several angular regions of the detector both in polar angle (end caps, transition region, barrel) and in azimuthal angle (to take into account non-instrumented zones in HCAL cracks), and (v) the very small number of dead ECAL channels (registered on-line) are taken into account and a correction is derived. Finally, complete systematic checks are made using low-energy lepton samples from $\gamma \gamma$ processes in addition to Bhabha and dimuon events. The misidentification of hadrons as leptons is investigated using pions tagged in $\tau \rightarrow \rho \nu_{\tau}$ decays by a reconstructed $\pi^{0}$. In the special case of hadrons misidentified as electrons, a new method using $d E / d x$ tagging is used to check the calorimeter-dependent part of the identification procedure.

\subsection{Selection of tracks and discriminating variables}

Some minimal cuts are necessary before a given particle track can be identified. Because of their range in HCAL, isolated muons can only be identified reliably above $1.8 \mathrm{GeV} / c$. Hence a minimum momentum value of $2 \mathrm{GeV} / c$ is required for muon and hadron candidates. The corresponding inefficiency is $5.0 \%$ for muons and $5.7 \%$ for hadrons. Since electrons can be well separated from heavier particles below $2 \mathrm{GeV} / c$ by $d E / d x$, no minimum momentum is imposed on them beyond the requirement of track reconstruction in the TPC $\left(p_{T}>\right.$ $150 \mathrm{MeV} / c)$. Finally a cut is applied around ECAL cracks for electrons and hadrons leading to an inefficiency of $4.7 \%$. These inefficiencies are known very precisely as they rely either on geometry or momentum calibration. The uncertainty from the momentum scale is estimated to be $10^{-4}$. The uncertainty in the muon momentum distribution due to $\tau$ polarisation is discussed in Section 6.2.

Eight variables are used in the identification procedure: $d E / d x$ in the TPC, two estimators of the shower profile in ECAL [12] ( $R_{T}$ for transverse shape and energy deposition, $R_{L}$ for longitudinal shape), the average shower width $\bar{W}$ measured with the HCAL tubes in the fired planes, the number of fired planes among the last ten $\left(N_{10}\right)$, the energy $E_{H}$ measured with HCAL pads, the number of hits $\left(N_{\mu}\right)$ in the muon chambers (within a road $\pm 4 \sigma$-wide around the track extrapolation, where $\sigma$ is the standard deviation expected from multiple scattering) and finally, the average distance $\bar{D}_{\mu}$ (in units of the multiple-scattering standard deviation) of the hits from their expected position in the muon chambers.

The correlation between discriminating variables is small except between $E_{H}$ and $\bar{W}$. In fact, $E_{H}$ was introduced to slightly improve $\mu$ - $h$ separation when a penetrating particle in a hadron shower causes an abnormally small width $\bar{W}$ and contributes significantly to the $N_{10}$ distribution. The $E_{H}$ variable is mainly used as a correction for this effect. No crack cuts are imposed for HCAL as the iron absorber configuration is azimuthally continuous. 
However special probability densities are used for tracks which extrapolate to the small noninstrumented areas between modules.

\subsection{Procedure and results}

The global $\mathrm{e} / \mu / h$ separation is applied to one-prong $\tau$ hemispheres and uses all the available variables. The reference distributions are checked against data using samples of known particles. Small discrepancies are observed in some distributions, in particular $R_{T}$ and $R_{L}$ for electrons. In the latter cases the distributions obtained from the electron data (see Section 3.4) are used when the identification is performed on $\tau$ data.

The identification efficiency matrix is first derived with the Monte Carlo simulation based on the KORALZ generator [13] for $\tau$-pair production. A significant improvement in performance is obtained compared to the earlier version used for the analysis of 1989-90 data [11]: the hadron misidentification to electron or muon is reduced by $40 \%$.

The momentum dependence of the electron and muon efficiencies is very weak. The hadron efficiency increases by $1 \%$ over the full momentum range due to the combined increase of hadron-to-electron and decrease of hadron-to-muon misidentifications.

A strong test of the validity of the method is obtained by looking at the distributions of the $P_{j}$ estimators for samples of identified particles in data and Monte Carlo. By construction one has $\sum P_{j}=1$ and in practice, due to $e$ - $\mu$ "orthogonality" in the detector, one of the following relations holds: $P_{e}+P_{h}=1$ or $P_{\mu}+P_{h}=1$. Therefore the chosen particle has always $P_{j}$ larger than 0.5 . The agreement is good (Fig. 1) over three orders of magnitude for all particle types. Indications of a slight overestimate of Monte Carlo efficiency are observed for muons and hadrons at the $10^{-3}$ level. Therefore the identification efficiencies are measured with data.

\subsection{Measurement of identification efficiencies}

The performance of the particle identification method is measured using samples of tagged particle types over the full momentum and angular ranges [14]. Bhabha events provide a high-energy electron sample $(>8 \mathrm{GeV} / c)$ and the $\gamma \gamma \rightarrow e^{+} e^{-}$process completes the range in the lower energy part. Similarly, a broad-band muon sample is obtained from $\mathrm{Z} \rightarrow \mu^{+} \mu^{-}$ and $\gamma \gamma \rightarrow \mu^{+} \mu^{-}$processes. In practice, lepton samples are obtained by tagging the opposite particle in each event with strict identification and momentum cuts. In all cases a small contribution from $\tau \tau$ events is subtracted in order to obtain the correct misidentification rates of leptons into hadrons. There are no suitable independent samples for hadrons, however $\tau$ decays into $\rho \nu_{\tau}$ where the $\pi^{0}$ from $\rho$ decay is reconstructed in ECAL, provide a sample of charged pions over the relevant kinematical range. 


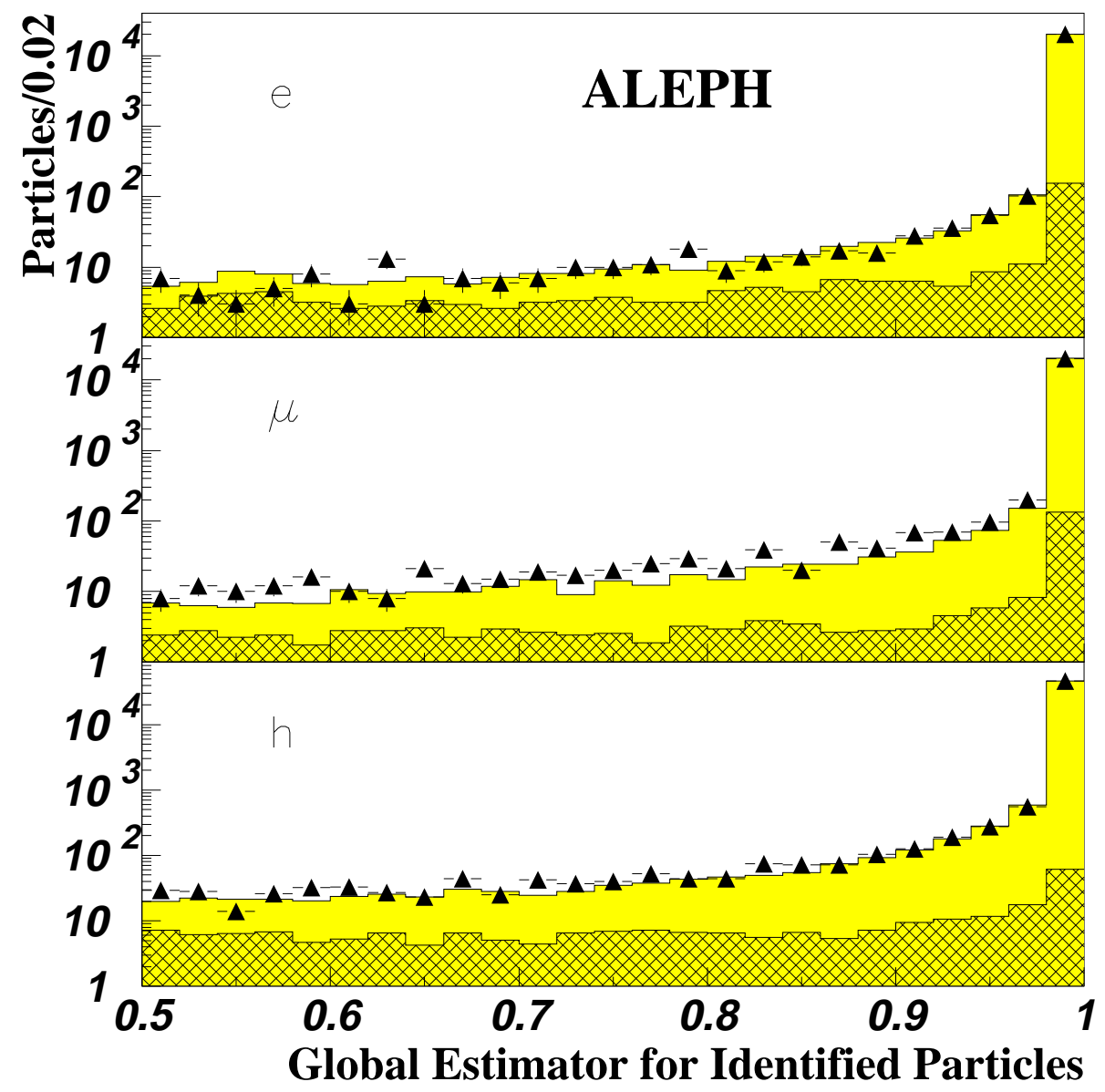

Figure 1: The distributions of the global estimators $\left(P_{e}, P_{\mu}\right.$, and $\left.P_{h}\right)$ for identified particles from $\tau$ decays. The plots show the data (triangles), the Monte Carlo (shaded histogram), and $\tau$ backgrounds (hatched histogram). The latter correspond to misidentified hadrons in the first two plots and misidentified leptons for the last plot. The hadronic veto defined in Section 5.2 is applied for electrons and muons in addition to the particle identification. 
The results of the comparison between data and Monte Carlo efficiencies is quite satisfactory for electrons and muons as shown in Fig. 2 as a function of momentum. The indication of a slightly smaller muon efficiency in data compared to Monte Carlo is confirmed here at the level anticipated from Fig. 1. The ratio of identification efficiencies in the independent lepton samples between data and Monte Carlo (Fig. 2) is used to renormalize the efficiency from the $\tau \tau$ Monte Carlo. In this procedure, the values for the ratio in the case of electrons are taken in each momentum bin, whereas for muons a linear fit is performed. This linear fit, in agreement with the measurements, is justified by the fact that the muons from $\mu \mu \gamma$ events are identified mostly in HCAL and the muon chambers, and do not interfere with the photon shower in ECAL. Furthermore, no fast variation of efficiency is expected for muons above $5 \mathrm{GeV} / c$. The situation is quite different for electrons in $e e \gamma$ events, because of the interplay between $d E / d x$ and ECAL estimators, and the presence of additional electromagnetic activity in ECAL. The experimental and Monte Carlo errors are then propagated and used as systematic uncertainties for the final identification efficiencies.

Angular dependence of the efficiencies is checked in a similar way. A small efficiency loss is observed in the barrel-end cap transition region for electrons (about 1\%) and is well reproduced by the simulation. A similar effect is seen in the azimuthal distribution of muons corresponding to the HCAL cracks, again well described by the Monte Carlo.

Hadron misidentification requires special attention because there are more hadrons than leptons and the simulation of their interactions in the calorimeters could be imperfect. The $\pi^{0}$ tagging method allows a detailed comparison of data and Monte Carlo for these specific final states, which are dominated by $\rho \rightarrow \pi \pi^{0}$ and $a_{1} \rightarrow \pi \pi^{0} \pi^{0}$, but also include a small contribution from $\mathrm{K}^{*} \rightarrow \mathrm{K} \pi^{0}$. The hadron sample selected in this way is therefore representative of the hadron contribution from $\tau$ decays. Fig. 3 shows good agreement for hadrons selected as muons or electrons, at least in the lower momentum range for the latter. Above $10 \mathrm{GeV} / c$ the Monte Carlo does not properly simulate hadron interactions in ECAL, particularly those interactions producing a large $\pi^{0}$ multiplicity inducing an electron-like shower. In order to check the interpretation of the effect, a complementary investigation is carried out using an independent hadron tagging using a tight $d E / d x$ cut in the TPC. This procedure allows a finalstate-independent study of the misidentification, regardless of the number of $\pi^{0}$ 's produced. The results obtained with this method are in excellent agreement with the previous ones: the excess of misidentification probability in data over Monte Carlo is $(1.8 \pm 0.5) \%$ with $\pi^{0}$-tagging and $(2.3 \pm 0.6) \%$ with the $d E / d x$ method. This confirms the discrepancy and supports its interpretation in terms of hadron interactions. The measured identification efficiency matrix is given in Table 1.

In summary, an accurate picture of particle identification is achieved in one-prong $\tau$ decays with data. Lepton efficiencies are measured with a systematic uncertainty of $1.0 \cdot 10^{-3}$ and hadron contamination is obtained with an uncertainty of $1.7 \cdot 10^{-3}$. It is however possible to further reduce the contaminations ( $2.5 \%$ in each lepton sample) using the information on additional photons in the lepton hemisphere as described in Section 5.2. Hence the respective 

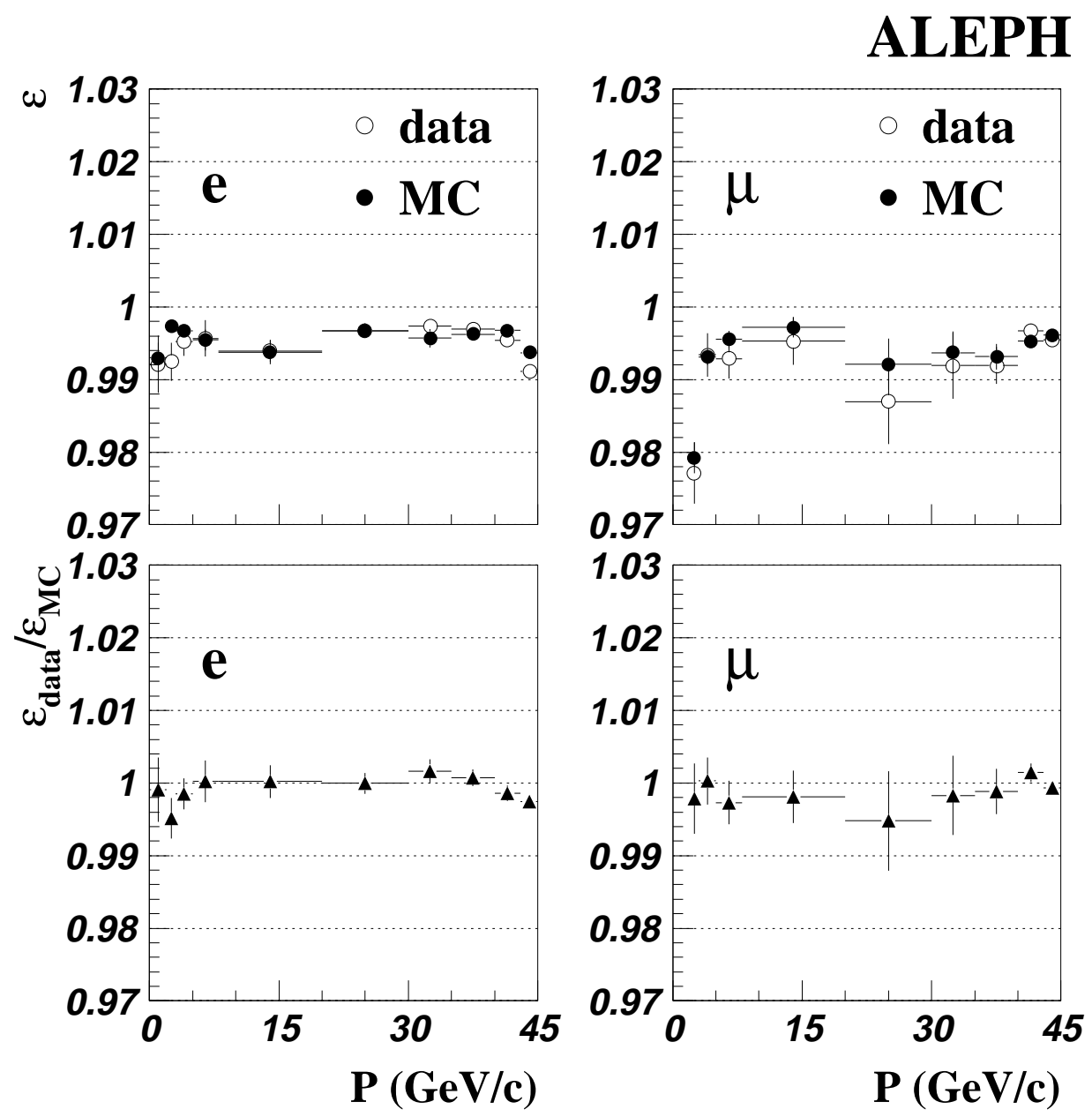

Figure 2: Identification efficiencies determined from the independent lepton samples and ratios between data and Monte Carlo efficiencies. The electron identification efficiency does not include the loss due to ECAL cracks. 


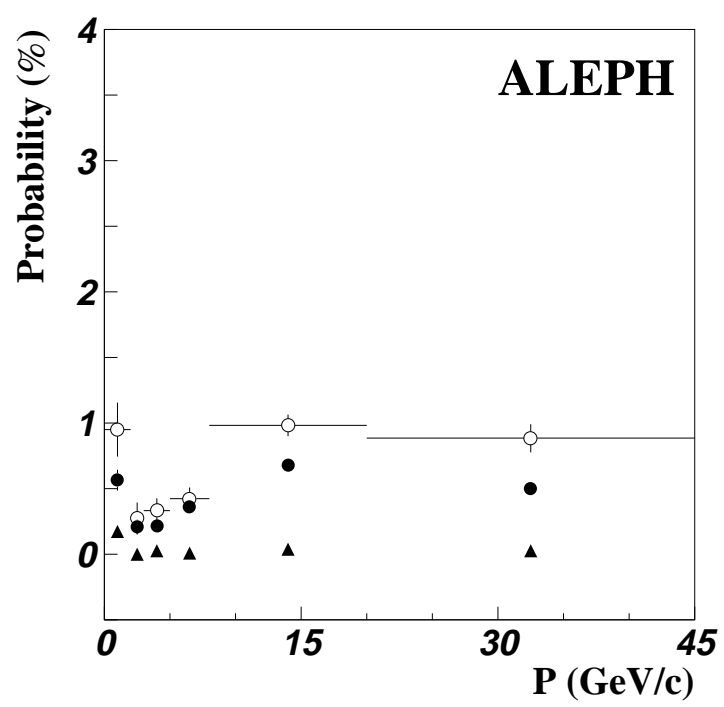

(a)

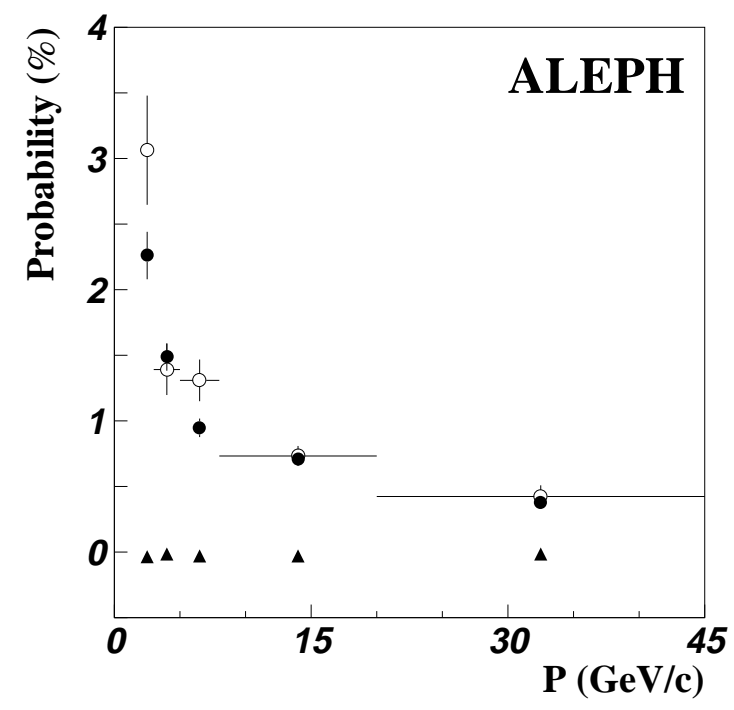

(b)

Figure 3: The misidentification probabilities of $\pi^{0}$-tagged hadron sample into leptons: (a) $h$ to $e$, (b) $h$ to $\mu$. The plots show the measurements with data (open circles), the full Monte Carlo contribution (black dots) and the specific component from leptonic decays with accidental $\pi^{0}$ tagging (triangles). 


\begin{tabular}{|c|r|r|r|}
\hline $\begin{array}{c}\text { true } \rightarrow \\
\downarrow \text { identified }\end{array}$ & \multicolumn{1}{|c|}{$e$} & \multicolumn{1}{c|}{$\mu$} & \multicolumn{1}{c|}{$h$} \\
\hline$e$ & $99.49 \pm 0.10$ & $<0.01$ & $0.79 \pm 0.06$ \\
\hline$\mu$ & $<0.01$ & $99.32 \pm 0.10$ & $0.90 \pm 0.06$ \\
\hline$h$ & $0.51 \pm 0.10$ & $0.68 \pm 0.10$ & $98.31 \pm 0.08$ \\
\hline
\end{tabular}

Table 1: Identification efficiency matrix (for charged particles above $2 \mathrm{GeV} / \mathrm{c}$ and not in ECAL cracks) on tagged samples (in \%).

systematic uncertainties will be further decreased.

\section{The selection of $\tau \tau$ events}

\subsection{Preselection procedure}

The principal characteristics of $\tau \tau$ events in $e^{+} e^{-}$annihilation are low multiplicity, backto-back topology and missing energy. Each event is divided into two hemispheres by a plane perpendicular to the thrust axis reconstructed by an energy flow algorithm [12] which calculates all the visible energy avoiding double-counting between the TPC and calorimeter information. The jet in a given hemisphere is defined by summing all the four-momenta of all energy flow objects (charged and neutral). The energies in the two hemispheres including the energies of photons from final state radiation, $E_{1}$ and $E_{2}$, are useful variables for separating Bhabha, $\mu \mu$ and $\gamma \gamma$-induced events from the $\tau \tau$ sample, while the relatively larger jet masses, wider opening angles, and higher multiplicities indicate $\mathrm{Z} \rightarrow q \bar{q}$ events. Each hemisphere is required to have at least one charged track. A charged track is defined to have at least four reconstructed space points in the TPC, to extrapolate well to the interaction point (within $\pm 2 \mathrm{~cm}$ transversally and $\pm 10 \mathrm{~cm}$ along the beams) and to satisfy $|\cos \theta|<0.95$.

All these features are incorporated in the standard $\tau \tau$ preselection used in ALEPH [15] and are briefly recalled in the following. Since the $\tau \tau$ events cannot be kinematically identified because of missing neutrinos, the philosophy of the method consists in reducing non- $\tau$ backgrounds to small levels without significantly biasing the $\tau \tau$ events.

Two-photon interactions are rejected by requiring an acollinearity angle between the two jets larger than $160^{\circ}$ while the sum of the jet energies is demanded to be larger than $0.35 E_{\text {beam }}$ or the difference between the transverse momenta of the two jets larger than $0.066 E_{\text {beam }}$. This reduces the $\gamma \gamma$ background to $0.3 \%$ at the $\mathrm{Z}$ peak energy.

To remove $\mathrm{Z} \rightarrow q \bar{q}$ events, the total charged-particle multiplicity is limited to 8 . If both hemispheres have more than one track or a jet mass larger than $1 \mathrm{GeV} / \mathrm{c}^{2}$, then the 
following conditions are imposed: (i) the product of the number of energy-flow objects in each hemisphere must be smaller than 75 and (ii) the sum of the maximum opening angles between two tracks in each hemisphere is smaller than $0.25 \mathrm{rad}$. The background from hadronic $\mathrm{Z}$ decays is thus reduced to $0.26 \%$, as calculated by the JETSET Monte Carlo [16]. As the remaining event configurations are quite atypical, checks are made comparing data and Monte Carlo distributions for enlarged cut values. Good agreement is found and a systematic uncertainty of $30 \%$ is estimated for this background.

Bhabha events must be treated with care because of their steep angular distribution and large cross section. The scattering angle $\theta^{*}$ in the $\tau \tau$ rest frame is calculated using the measured polar angle $\theta_{+}\left(\theta_{-}\right)$of the positively (negatively) charged jet through the relation

$$
\cos \theta^{*}=\frac{\sin \left(\frac{\theta_{+}-\theta_{-}}{2}\right)}{\sin \left(\frac{\theta_{+}+\theta_{-}}{2}\right)} .
$$

A cut $\left|\cos \theta^{*}\right|<0.90$ defines the angular acceptance for the $\tau \tau$ sample while rejecting smallangle Bhabha events. For Bhabha-like events (where all charged particles are identified as electrons with loose criteria) the normalized total energy, $x_{\text {tot }}=E_{\text {tot }} / E_{\text {beam }}$, is required to be smaller than 1.6 (or 1.4 if the tangent to the leading electron points to within $\pm 6 \mathrm{~cm}$ of an ECAL crack). Here $E_{\text {tot }}$ includes the energy carried away by a radiative photon assumed to be emitted along the beam line; its energy is calculated using three-body kinematics. All this reduces the Bhabha contamination to $0.66 \%$ at peak energy, by far the largest background.

Finally, $Z \rightarrow \mu \mu$ events are rejected by the requirement that the sum of the momenta of the two leading tracks is less than $1.6 E_{\text {beam }}$. Also, for loosely defined dimuon events, $x_{\text {tot }}$ must be smaller than 1.8 . The resulting $\mu \mu$ background is $0.25 \%$.

\subsection{Further selection using the likelihood particle identification}

The total contamination after preselection is $1.78 \%$ at peak energy, including $0.14 \%$ from four-fermion processes ${ }^{1}$ and $0.17 \%$ from cosmics (reduced in the preselection by tightening the pointing cuts to the interaction region for at least one track). These results are improved [14] using the particle identification described in Section 3.

Particular attention is paid to the complete event topology to reduce the Bhabha background. For example, the track opposite to an identified electron could go through an ECAL crack and hence would not be identifiable as an electron. Also $e-h$ topologies are

\footnotetext{
${ }^{1}$ The $\gamma \gamma$-processes mentioned above also involve four fermions, but are dominated by configurations with two leptons in the detectors while electrons and positrons stay in the beam pipe. The four-fermion processes refer here to high $q^{2}$ events enhanced by the $\mathrm{Z}$ pole giving final states with three of four leptons at large angle to the beams. The overlap between the two samples is negligible [17].
} 
considered taking into account $e \rightarrow h$ misidentification. Additional cuts are applied in the $E_{1}, E_{2}$ plane depending on the event topology and according to the $\cos \theta^{*}$ range. In particular, events where both sides are identified as electrons are rejected for $\cos \theta^{*}>0.7$ if $E_{1}>40 \mathrm{GeV}$ and $E_{2}>10 \mathrm{GeV}$ or vice versa (Fig. 4). Other topologies $\left(e-e\right.$ with $\cos \theta^{*}<0.7, e$-crack, $\left.e-h\right)$ are treated in a similar way.
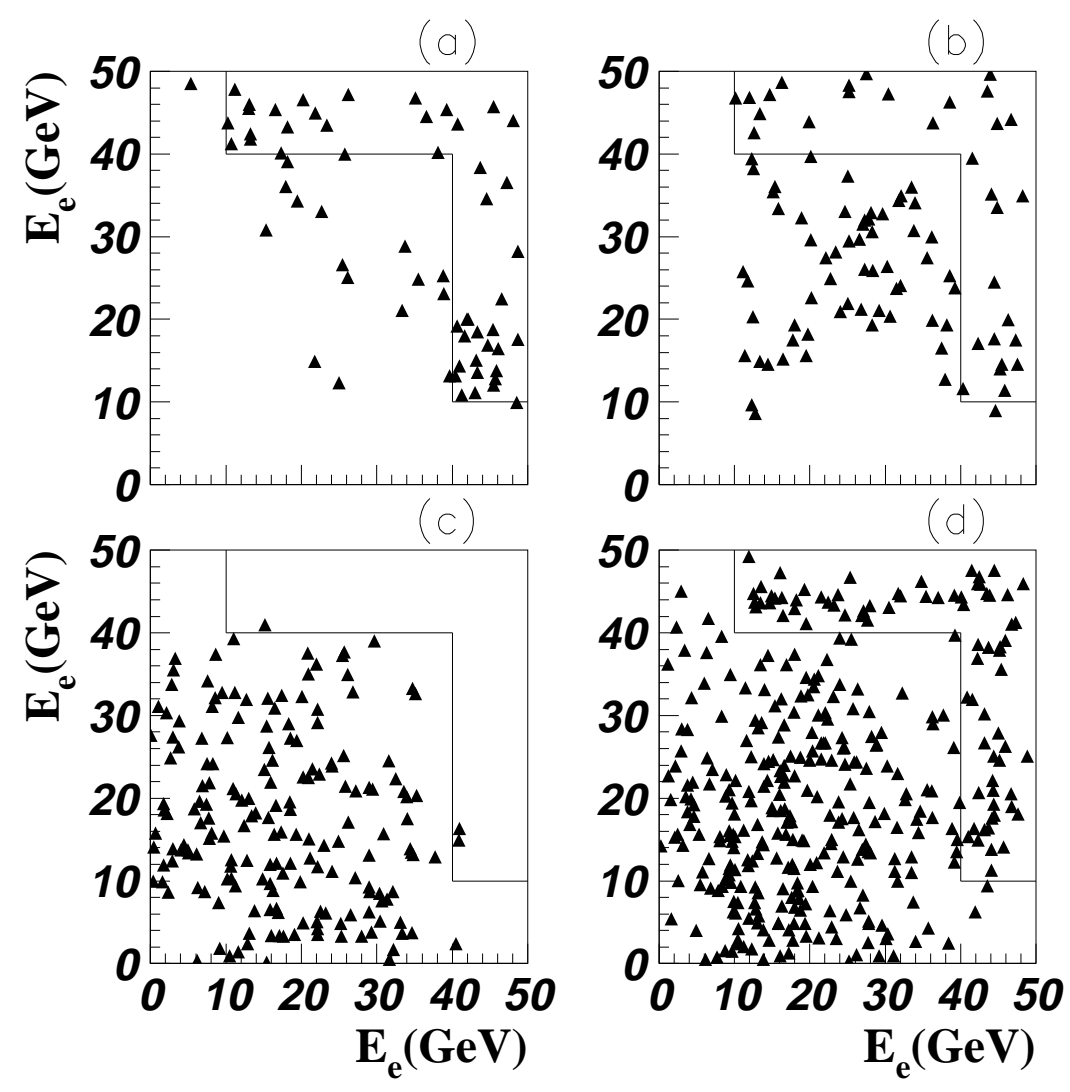

Figure 4: The energy distributions for e-e events with $\cos \theta^{*}>0.7$ after preselection. The plots correspond to samples of Bhabha Monte Carlo (BABAMC [18] (a) and UNIBAB [19] (b)), $\tau \tau$ Monte Carlo (c) and data (d). The samples are not normalized to the same luminosity.

Finally a last cut is applied on $x_{\text {tot }}$ and the acoplanarity $\Delta \phi$ between the two jets: events are rejected if $\Delta \phi<1^{\circ}$ and $x_{\text {tot }}>1.2$ for $\left|\cos \theta^{*}\right|>0.7$ (Fig. 5), and, $\Delta \phi<0.4^{\circ}$ and $x_{t o t}>1.5$ for $\left|\cos \theta^{*}\right|<0.7$.

These additional cuts decrease the $\tau \tau$ efficiency by only $0.13 \%$ while reducing the Bhabha contamination by a factor of 5 . The estimate of the final contamination is given below. 


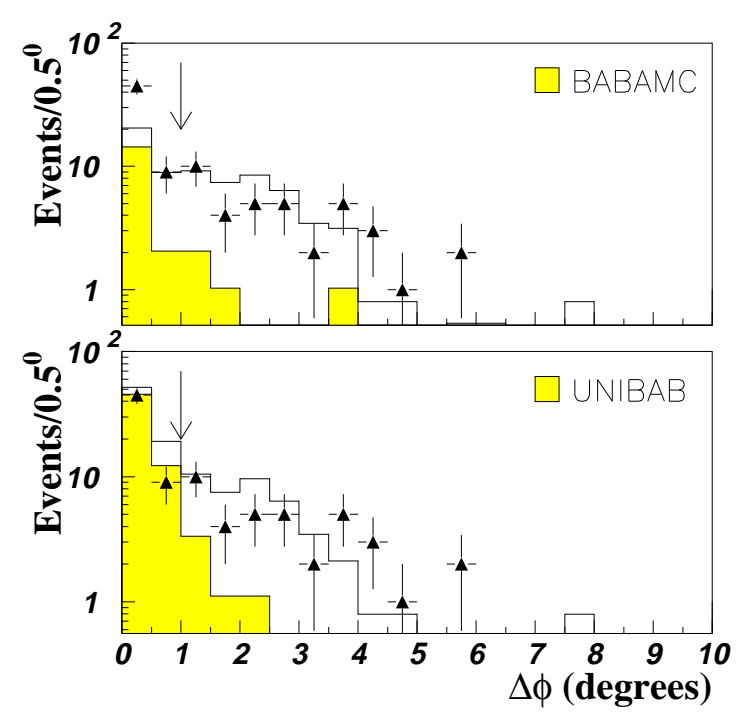

(a)

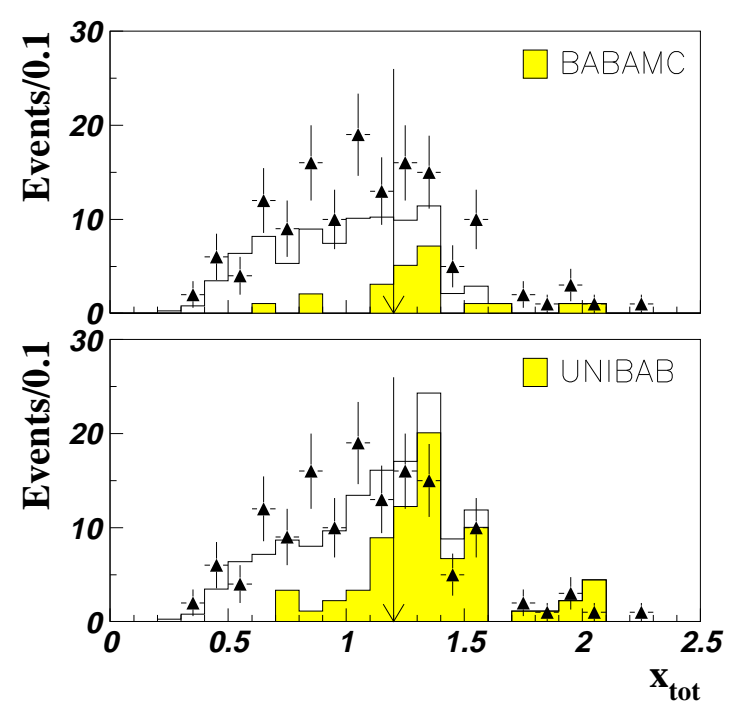

(b)

Figure 5: (a) The acoplanarity distributions for e-e topology where $\left|\cos \theta^{*}\right|>$ 0.7 and $x_{\text {tot }}>1.2$ are already required. (b) The total energy distributions for e-e topology with $\left|\cos \theta^{*}\right|>0.7$ and $\Delta \phi<1^{\circ}$. Data are shown in triangles and the histograms correspond to the $\tau \tau$ Monte Carlo and the Bhabha Monte Carlo (shaded). The arrows indicate the location of the respective cuts. The agreement with data is better with the UNIBAB Bhabha Monte Carlo (see Section 4.3). 
Similarly the $\mathrm{Z} \rightarrow \mu \mu$ events can be further reduced: if both tracks are identified as muons and their momenta are larger than $10 \mathrm{GeV} / c$ and $43 \mathrm{GeV} / c$, then the event is rejected (Fig. 6). Slightly tighter cuts are used for $\mu$ - $h$ topologies.

Additional cuts are also applied against $\gamma \gamma$-induced processes, restricting further the acollinearity condition $\left(>170^{\circ}\right)$ for $e-e$ and $\mu-\mu$ final states with small energies and small momentum asymmetries between the two hemispheres.

Cosmic ray background can be reduced to a negligible level by using the very tight matching between the "two" tracks in space and in momentum. Most of the contamination can be found in the $\mu$-X topology with a small number of ITC hits since cosmics are not in time with the beam crossing (Fig. 7 (a), (b)). The complementary sample, $\mu-X$ events with a larger number of ITC hits and $h$ - $h$ events with any number of ITC hits, is composed of mostly $\tau \tau$ events, almost in-time cosmics $(\mu-X)$ and grossly out-of-time cosmics $(h-h)$ where the misidentification results from the small efficiency of the HCAL streamer tubes in these conditions (Fig. 7 (c)). The cosmic background can thus be overdetermined for the most part and the final contamination is estimated to be $(0.024 \pm 0.003 \%)$ with no loss of $\tau \tau$ efficiency.

\subsection{Estimate of remaining backgrounds}

Apart from cosmic ray background which is almost completely removed, and contamination from $\mathrm{Z} \rightarrow q \bar{q}$ (discussed in Section 4.1) and four-fermion processes which is estimated from Monte Carlo generators, respectively from Ref. [16] and Ref. [20], all other backgrounds should be treated with caution because they are possibly not well described either at the generator level or at the level of the ALEPH detector simulation. In order to study these problems, extensive comparisons of data and different Monte Carlo programs with detector simulation are performed.

Two generators are used for studies of Bhabha background: BABAMC [18] with initial and final state radiation to first order, and UNIBAB [19] with first-order final state radiation and incorporating higher order corrections in the initial state radiation through exponentiation. Better agreement is generally found with UNIBAB especially for the acollinearity distribution. In order to estimate the background remaining after all cuts, the rejection rates in data and Monte Carlo are monitored at the level of every cut, the last applied being the more closely related to the final contamination. The final estimate of the remaining background for each generator is given by

$$
N_{B}=N_{B M C}^{l e f t} \cdot\left(\frac{N_{\text {data }}-N_{\tau \tau M C}}{N_{B M C}}\right)_{\text {rejected by lastcuts }},
$$

where $N_{B}$ is the estimated number of remaining background events for the process labeled $B, N_{B M C}^{l e f t}$ is the corresponding value for the $B$ Monte Carlo and the term in parentheses 


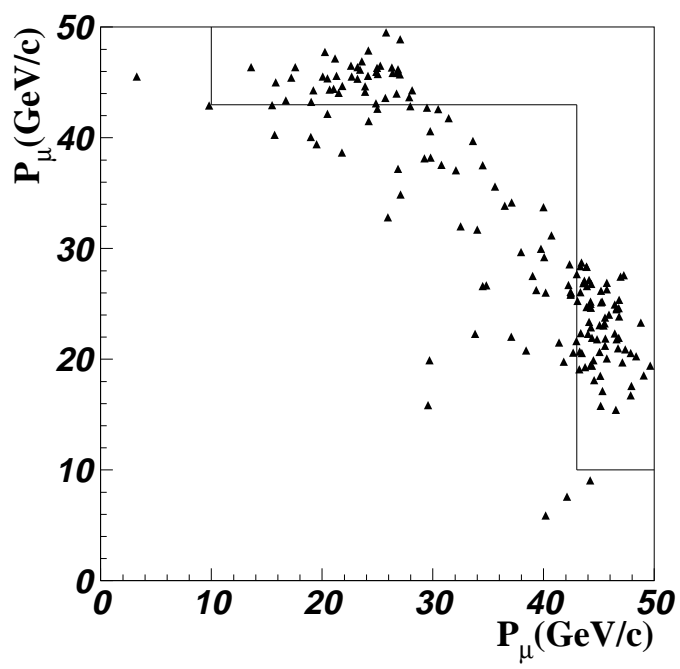

(a)

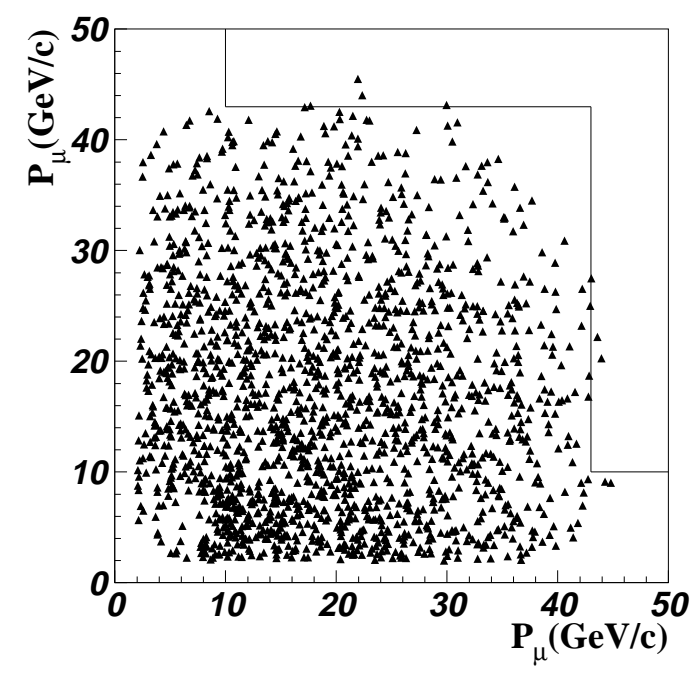

(b)

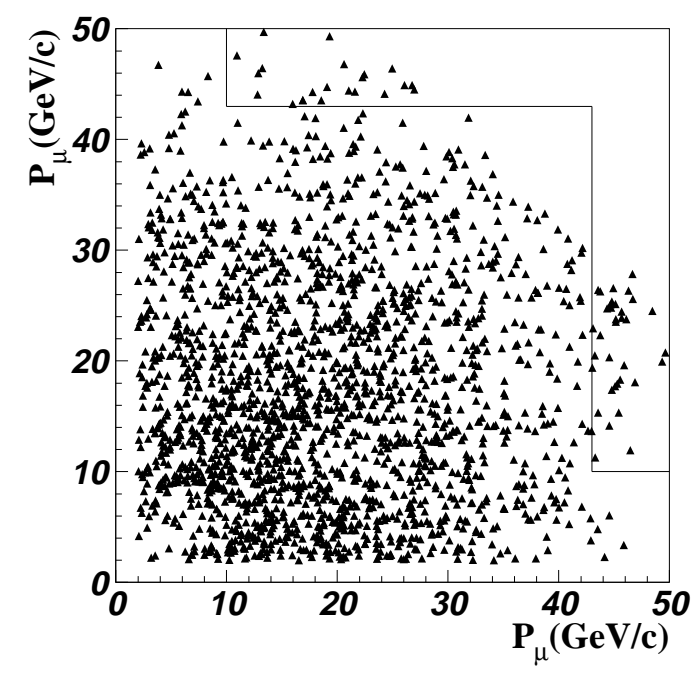

(c)

Figure 6: The momentum distributions for $\mu-\mu$ events after preselection. The plots correspond to samples of (a) $e e \rightarrow \mu \mu$ Monte Carlo, (b) $\tau \tau$ Monte Carlo and (c) data. The samples are not normalized to the same luminosity. 


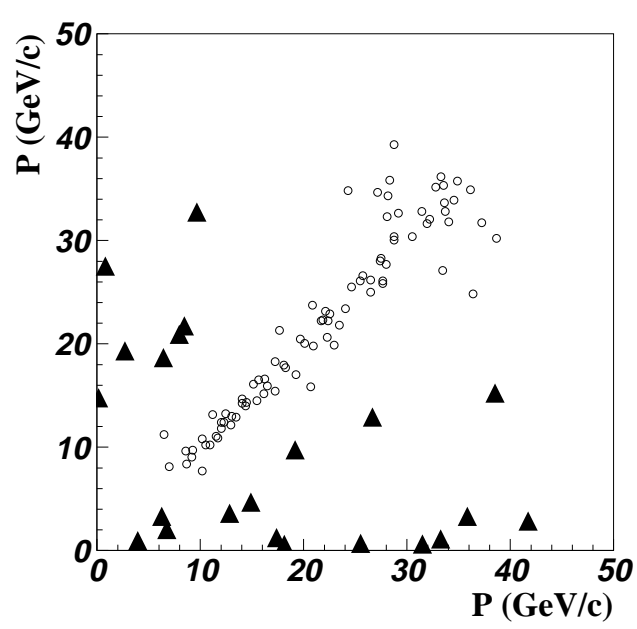

(a)

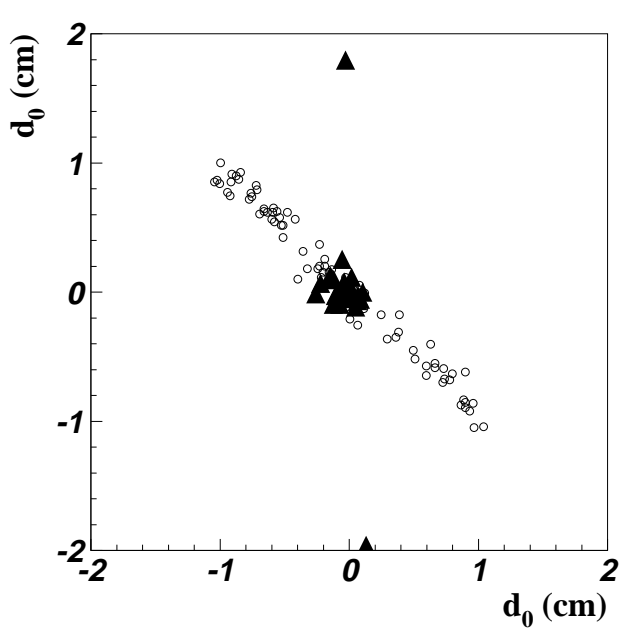

(b)

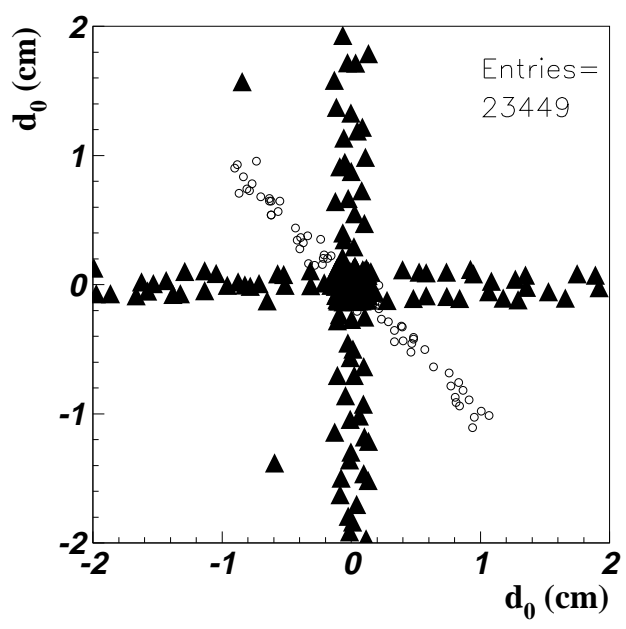

(c)

Figure 7: The cosmic ray rejection after preselection for $\mu$-X events with fewer than five ITC hits for one track and fewer than six for the other, out of a maximum of eight hits per track. The plots show the correlations between the two tracks for (a) momentum and (b) transverse distance of approach to the interaction point. The transverse distance $d_{\circ}$ is given a sign depending on the track angular momentum. The few events which do not show momentum balance are kept as $\tau \tau$ candidates (triangles) while the others are cosmic rays (open circles). The last plot (c) corresponds to the complementary sample dominated by $\tau \tau$ events clustered at small $d_{\circ}$ values with tails due to bremsstrahlung and interactions in the detector. The cosmic contamination in this sample is evident. 
refers to the number of events rejected by the cuts applied after the preselection in the data, the $\tau \tau$ Monte Carlo and the $B$ background Monte Carlo. In other words, the final estimate relies on the shape of the Monte Carlo distributions of the variables used in the last cuts while the absolute rate is normalized by the actual rejection rate of these cuts. The shapes of the $E_{1,2}, \Delta \phi$, and $E_{\text {tot }}$ distributions are in good agreement between the two generators, leading to consistent estimates for the remaining background. The consistency between the two estimates is a test of the robustness of the method for estimating the background. This comparison provides an estimate of the systematic effects related to the Bhabha Monte Carlo generators, included in the final uncertainty on the non- $\tau$ backgrounds.

A similar approach is taken for the other sources of background. In all cases the procedure leads to an estimate of the background which does not depend on the absolute Monte Carlo prediction. For Bhabha and $\mathrm{Z} \rightarrow \mu \mu$ events, the estimates are significantly different from the Monte Carlo predictions, a fact which is not surprizing considering the large rejection rate achieved and the difficulty to properly simulate the detector performance at this level. For $\gamma \gamma$-induced processes however, the agreement between the estimate from data and the Monte Carlo prediction is good (data/Monte Carlo $=1.03 \pm 0.26$ for $\gamma \gamma e e$ and $0.86 \pm 0.20$ for $\gamma \gamma \mu \mu$ ) which is expected since the rejection is achieved essentially through kinematics and does not depend crucially on detector resolution. The systematic uncertainties introduced by the cuts which depend on energy calibration and resolution are discussed in Section 6.

The final contaminations with their systematic uncertainties are given in Table 2 for the selected $\tau \tau$ events and for the identified lepton samples defined more precisely in Section 5 . The contamination in the $\tau \tau$ sample is $(0.88 \pm 0.09) \%$ for the full data set. The background is reduced by a factor of 2.2 compared to the preselection step at the expense of a total loss of $0.19 \%$ in $\tau \tau$ efficiency.

\begin{tabular}{|l|ccc|}
\hline & $\tau \tau$ & $e$ & $\mu$ \\
\hline Bhabha & $0.15 \pm 0.03$ & $0.49 \pm 0.08$ & 0 \\
cosmic rays & 0.02 & 0 & 0.01 \\
$\mathrm{Z} \rightarrow \mu \mu$ & $0.07 \pm 0.02$ & 0 & $0.35 \pm 0.09$ \\
$\gamma \gamma$ processes & $0.23 \pm 0.03$ & $0.46 \pm 0.10$ & $0.47 \pm 0.10$ \\
$\mathrm{Z} \rightarrow q \bar{q}$ & $0.26 \pm 0.08$ & 0 & 0 \\
four-fermion & $0.14 \pm 0.02$ & $0.22 \pm 0.02$ & $0.07 \pm 0.02$ \\
\hline sum & $0.88 \pm 0.09$ & $1.18 \pm 0.14$ & $0.90 \pm 0.14$ \\
\hline
\end{tabular}

Table 2: Summary of non- $\tau$ backgrounds in the final samples (in \%). 


\subsection{Measurement of selection efficiencies}

Apart from simple cuts defining the geometrical acceptance which are not affected by significant systematics, the selection depends on energy cuts which are sensitive to the simulation of the detector and must therefore be carefully evaluated. Inefficiencies induced by the major cuts are given in Table 3 for the different $\tau \tau$ topologies.

\begin{tabular}{|c|c|c|c|c|}
\hline cause for inefficiency & $\tau \tau$ & $e-X$ & $\mu-X$ & $h-h$ \\
\hline \multicolumn{5}{|c|}{ geometrical acceptance and multiplicity } \\
\hline $\left.\begin{array}{l}\text { Number of good tracks }<2 \text { or }>8 \\
\left|\cos \theta^{*}\right|>0.9\end{array}\right\}$ & 16.51 & 17.14 & 16.04 & 16.36 \\
\hline \multicolumn{5}{|l|}{ preselection } \\
\hline Acollinearity $<160^{\circ}$ & 1.07 & 1.70 & 1.54 & 0.41 \\
\hline$E_{1}+E_{2}<0.35 \cdot E_{\text {beam }}$ when $\delta P_{T}<0.066 E_{\text {beam }}$ & 1.10 & 2.17 & 2.19 & 0.15 \\
\hline$N_{o b j 1} \cdot N_{o b j 2}>75$ & 0.16 & 0 & 0 & 0.38 \\
\hline Maximum opening angle sum $>0.25 \mathrm{rad}$ & 1.61 & 0.39 & 0.30 & 3.30 \\
\hline Leading energy sum $>1.6 E_{\text {beam }}$ & 0.55 & 0.28 & 0.39 & 0.87 \\
\hline$x_{\text {tot }}$ cut & 1.23 & 1.40 & 0.85 & 1.22 \\
\hline \multicolumn{5}{|l|}{ additional cuts } \\
\hline Bhabha rejection & 0.13 & 0.34 & 0 & 0.05 \\
\hline$\gamma \gamma$ rejection & 0.03 & 0.06 & 0.04 & 0 \\
\hline $\mathrm{Z} \rightarrow \mu \mu$ rejection & 0.03 & 0 & 0.08 & 0 \\
\hline total inefficiency & 21.32 & 22.26 & 20.47 & 21.58 \\
\hline
\end{tabular}

Table 3: Monte Carlo values for the inefficiencies at peak energy (in \%). $h$ - $h$ is the event topology with both non-leptonic hemispheres. $X$ corresponds to any $\tau$ decay final state.

Every cut has been examined in turn. The corresponding distributions from data and Monte Carlo are compared before the cut is applied and the amount of data rejected by the cut is fitted, using the simulated distribution for the backgrounds. The latter is normalized at an appropriate place in order to minimize the dependence on the simulation of the resolution. As an example, Fig. 8 illustrates this procedure for the $x_{\text {tot }}$ cut used to reject Bhabha events. The efficiency of the cut on data is determined with a statistical error from the data and Monte Carlo samples, and a systematic error reflecting the uncertainty on the background subtraction, obtained by changing the normalization point. This procedure applied to the $x_{\text {tot }}$ cut (Fig. 8) yields a ratio of data to Monte Carlo efficiencies differing from unity by $(0.80 \pm 0.55($ stat $) \pm 0.33($ syst $)) \cdot 10^{-3}$. In all cases, corrections are applied to the Monte Carlo efficiencies and the errors are added in quadrature to obtain the systematic uncertainty. 


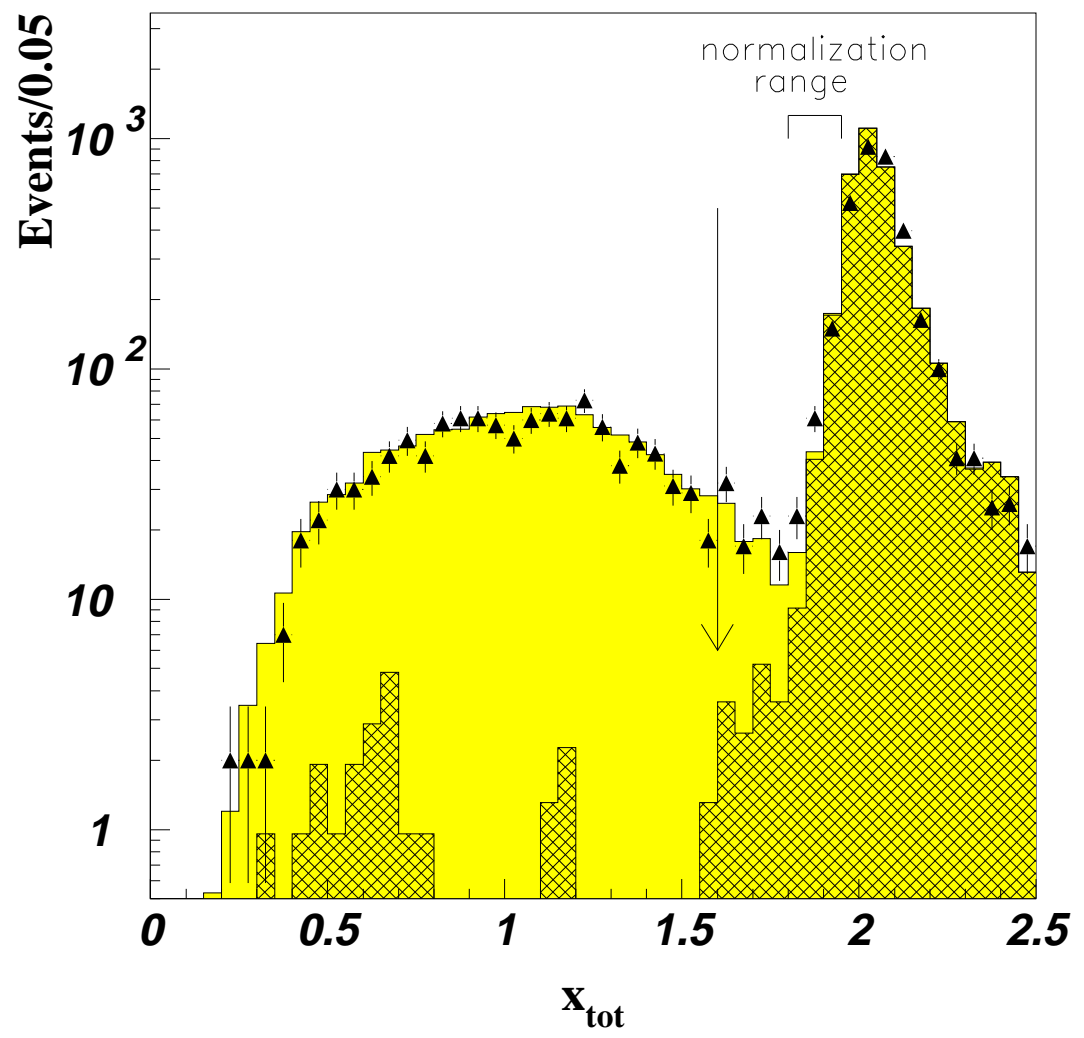

Figure 8: $x_{\text {tot }}$ distributions for lepton events where the events are flagged as Bhabha-like. The shaded histogram corresponds to the $\tau \tau$ Monte Carlo, whereas the hatched histogram represents the simulated contribution of Bhabha events generated with UNIBAB normalized in the three marked bins. The vertical arrow corresponds to the location of the applied cut. 
Taking into account all cuts applied to remove leptonic backgrounds, the measured corrections to the Monte Carlo efficiencies are found to be

$$
\begin{aligned}
& \frac{\Delta \varepsilon_{e^{-X}}}{\varepsilon_{e^{-} X}}=(-0.7 \pm 0.9) \cdot 10^{-3} \\
& \frac{\Delta \varepsilon_{\mu^{-X}}}{\varepsilon_{\mu^{-X}}}=(-0.1 \pm 0.7) \cdot 10^{-3}
\end{aligned}
$$

for the leptonic samples $e-X$ and $\mu-X$ where $X$ is any $\tau$ final state.

Although the leptonic cuts are well reproduced by simulation, the situation is less satisfactory concerning the hadronic cuts. Low energy calorimeter clusters are not simulated accurately, leading to discrepancies in energy-flow variables, particularly the jet mass and the number of calorimeter objects. Assuming only that the two hemispheres are not correlated for these two variables, it is possible to determine the efficiency of the corresponding cuts by constructing unbiased jet samples both in data and Monte Carlo. The Monte Carlo sample is used to evaluate the small correlations and to correct the result. The effect of all cuts affecting hadronic final states (both leptonic and hadronic cuts as shown in Table 3 ) is evaluated, revealing a small but significant discrepancy between data and Monte Carlo:

$$
\frac{\Delta \varepsilon_{h-h}}{\varepsilon_{h-h}}=(-12.1 \pm 2.0) \cdot 10^{-3}
$$

The correction for hemisphere correlations mentioned above is $(-2.0 \pm 1.0) \cdot 10^{-3}$, and is included in the final value.

Taking all final states into consideration, the $\tau \tau$ selection efficiency at the $\mathrm{Z}$ peak is found to be $\varepsilon_{\tau \tau}=(78.27 \pm 0.12) \%$, where the uncertainty is dominated by the statistical error of the data-Monte Carlo comparison. Other uncertainties can also affect $\varepsilon_{\tau \tau}$ : in particular, triggering and tracking could produce systematic effects not well simulated by Monte Carlo. Studies have shown that the corresponding uncertainty is $0.12 \%$. However it does not contribute appreciably to this analysis as $l-X$ and all $\tau \tau$ final states are affected in a similar way because of the dominance of one-prong decays.

It should be emphasized that although the $\tau \tau$ Monte Carlo has been used as a reference in this determination, the efficiency values are corrected with measurements from the data, including detector effects not necessarily included in the simulation and possible deficiencies at the generator level such as radiative effects, for example. The "geometrical" part of the acceptance is calculated with the Monte Carlo which incorporates initial and final state radiation; the corresponding statistical error $(0.092 \%)$ is included in the final uncertainty on $\varepsilon_{\tau \tau}$.

The statistics for the selected $\tau \tau$ sample is summarized in Table 4. 


\begin{tabular}{|l|c|}
\hline$N_{\tau \tau}$ & 62249 \\
\hline$\varepsilon_{\tau \tau}$ & $0.7814 \pm 0.0012$ \\
$f_{\tau \tau}^{\text {non- } \tau}$ & $0.0088 \pm 0.0009$ \\
\hline
\end{tabular}

Table 4: Summary of the $\tau \tau$ data sample for 1991-93. $\varepsilon_{\tau \tau}$ is the $\tau \tau$ selection efficiency determined from data as explained in Section 4 taking into account peak and off-peak samples, and $f_{\tau \tau}^{n o n-\tau}$ is the estimated contamination from non- $\tau$ backgrounds.

\section{$5 \quad$ Final definition of the leptonic decays}

The likelihood particle identification described in Section 3 relies only on measurements of the charged particle. Considering now the complete hemisphere with a lepton candidate, some final improvements in the classification are introduced in order to reduce systematic effects at the level of $10^{-3}$. In addition, it should be pointed out that no requirement is imposed on the number of photons in the lepton hemisphere.

\subsection{Requirement of a single track}

About $2 \%(0.4 \%)$ of $\tau$ decays to electrons (muons) have more than one track because of photons from radiation or bremsstrahlung converting into $e^{+} e^{-}$pairs. An analysis with no restriction on the number of tracks would have to reconstruct the converted photons and hence the original topology. This procedure is difficult to test at the $10^{-3}$ level and furthermore background introduced from hadronic channels (for a given $h \rightarrow e$ misidentification) would be significantly increased. To avoid these problems only hemispheres with a single track are selected in this analysis.

The problem still remains to experimentally determine the probability for a leptonic decay to appear with a single lepton track. This is achieved by using the Monte Carlo efficiency, corrected appropriately from detailed comparisons with data. A first method uses the distribution of the number of reconstructed tracks without a strict requirement that the extrapolated tracks pass near the interaction region (most of the $e^{+} e^{-}$pairs produced outside the beam pipe with small momenta do not yield good tracks in the restricted definition). A second method checks in a first step the photon multiplicity detected in ECAL for lepton hemispheres; in a second step the conversion rate leading to at least one good reconstructed track is normalized using a detailed comparison between data and Monte Carlo for the $\rho \rightarrow \pi \pi^{0}$ sample of $\tau$ decays where one of the photons converts into a pair.

The two independent methods give consistent estimates [14] for the rate of lepton hemispheres with more than one track. They are averaged to yield $(1.83 \pm 0.07) \%$ for electrons 
and $(0.35 \pm 0.03) \%$ for muons whilst the simulation predicts $(1.69 \pm 0.05) \%$ and $(0.36 \pm 0.02) \%$, respectively.

\subsection{Hadronic veto}

So far particle identification only uses information related to the candidate particle, leading to hadronic contamination of about $2.5 \%$, which is known with an uncertainty of $0.17 \%$. Fortunately at least $75 \%$ of one-prong hadronic $\tau$ decays are expected to include $\pi^{0}$ 's which can be detected in ECAL. More precisely, cuts can be applied on the hadronic invariant mass (assigning the pion mass to the lepton candidate, $\left.M_{\pi \gamma . . \gamma}\right)$ and the "photonic" mass $\left(M_{\gamma . . \gamma}\right)$ using all photon candidates in the lepton hemisphere. These cuts define a hadronic veto which overrides the lepton identification from the likelihood method.

For muons, the hadronic veto is straightforward as photons are clearly detected without interference from the minimum-ionizing particle: hemispheres with $M_{\gamma . . \gamma}>0.1 \mathrm{GeV} / c^{2}$ and $0.5<M_{\pi \gamma . . \gamma}<1.4 \mathrm{GeV} / c^{2}$ are rejected. This cut removes a clear hadron signal, dominated by the $\rho \rightarrow \pi \pi^{0}$ and $a_{1} \rightarrow \pi \pi^{0} \pi^{0}$ channels which is well reproduced by the Monte Carlo (Fig. 9).

A similar procedure is applied to electrons. However the presence of photons in a hadronic decay interferes with the charged track identification and the values for the cuts are adjusted depending on the likelihood probability of the lepton candidate. Fig. 10 shows the hadronic mass distribution with a clear hadronic contamination. Here the simulation underestimates the overall misidentification as already discussed in Section 3. In most cases of hadrons misidentified as electrons, one energetic photon from a $\pi^{0}$ is merged into the ECAL shower associated to the pion and the shower appears more "electromagnetic". For this reason the $M_{\gamma . . \gamma}$ mass distribution is less useful than for muons and it is simpler to rely on $M_{\pi \gamma . . \gamma}$ alone $\left(M_{\pi \gamma . . \gamma}>0.5 \mathrm{GeV} / c^{2}\right)$.

After these cuts, the hadron contamination is reduced by a factor of 1.9 for electrons and 2.4 for muons with corresponding efficiency losses of $0.07 \%$ and $0.04 \%$, respectively. The systematic uncertainty on the lepton efficiency is smaller than $10^{-3}$, including an estimate for the effect of neglected QED higher orders in the radiative corrections for the $\tau \tau$ final states and $\tau$ decays. The momentum dependence of the final contaminations is given in Fig. 11 . Averaging over the hadron spectrum, this gives $(1.26 \pm 0.07) \%$ for electrons and $(1.11 \pm 0.08) \%$ for muons.

\section{Additional systematic studies}

The most important sources of systematics $-\tau \tau$ selection, lepton identification, and hadron contamination-have been discussed in the preceding sections. In this section, additional 


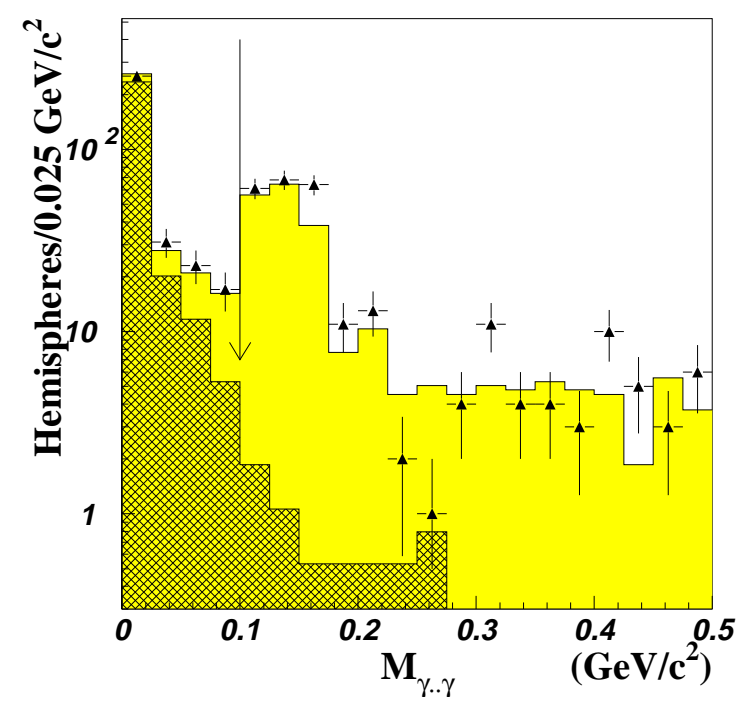

(a)

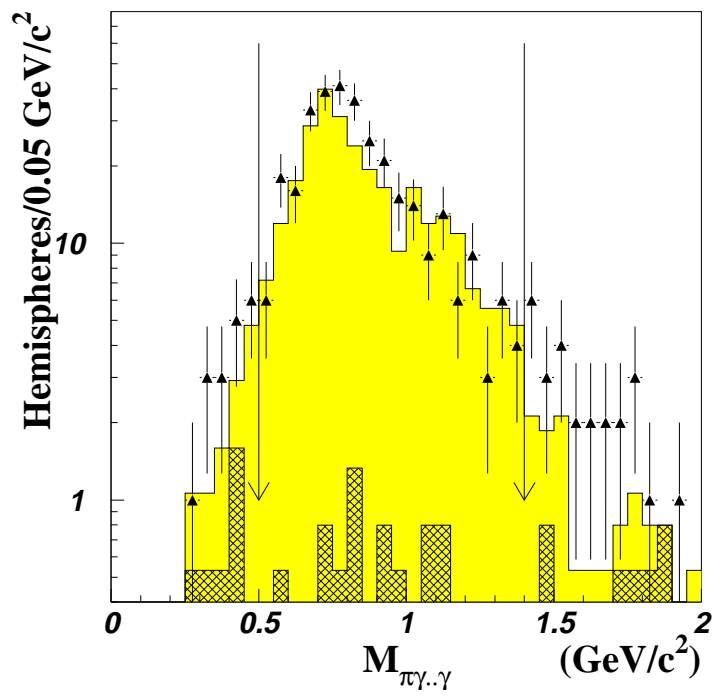

(b)

Figure 9: $M_{\gamma \cdot \cdot \gamma}$ and $M_{\pi \gamma \cdot \cdot \gamma}$ distributions to veto hadronic final states in which the particle is identified as a muon. (a) $M_{\gamma \cdot \cdot \gamma}$ after $M_{\pi \gamma \cdot \cdot \gamma} c u t$, (b) $M_{\pi \gamma \cdot \gamma}$ after $M_{\gamma \cdot \gamma}$ cut. The arrows show the cuts to further purify the muon sample. Data are shown as triangles. The shaded histogram corresponds to Monte Carlo $\tau$ decays where the charged hadron is misidentified as a muon, while the hatched histogram represents the contribution from muonic $\tau$ decays with at least one photon. 


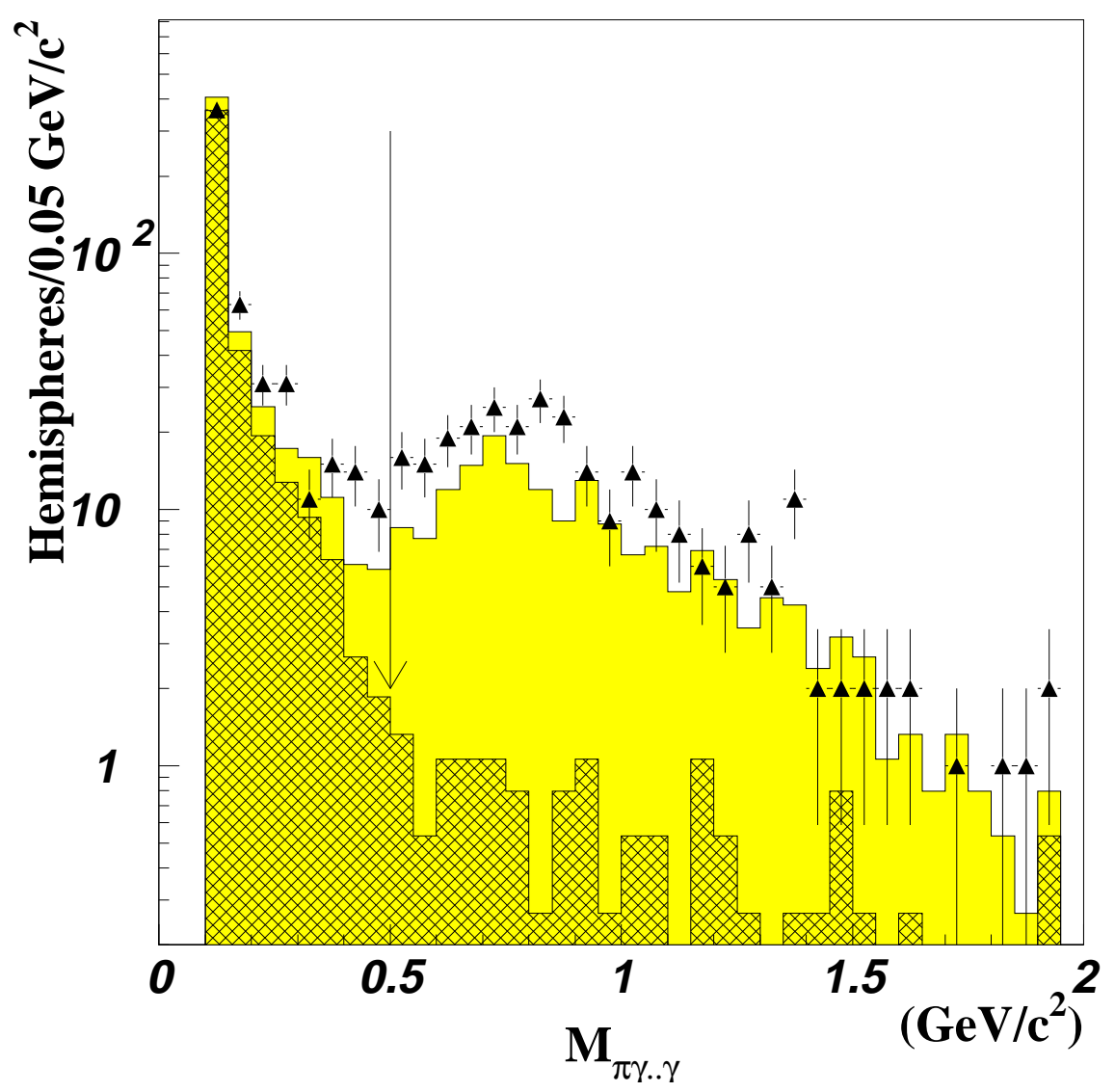

Figure 10: $M_{\pi \gamma \cdot \gamma}$ distribution to veto hadronic final states in which the particle is identified as an electron. Data are shown as triangles and the shaded histogram corresponds to Monte Carlo $\tau$ decays where the charged hadron is misidentified as an electron. The hatched histogram shows the Monte Carlo contribution from electronic $\tau$ decays with at least one photon. The arrow shows the cut on this mass to further purify the electron sample. The discrepancy between data and Monte Carlo for the rate of hadron-to-electron misidentification is discussed in Section 3. 

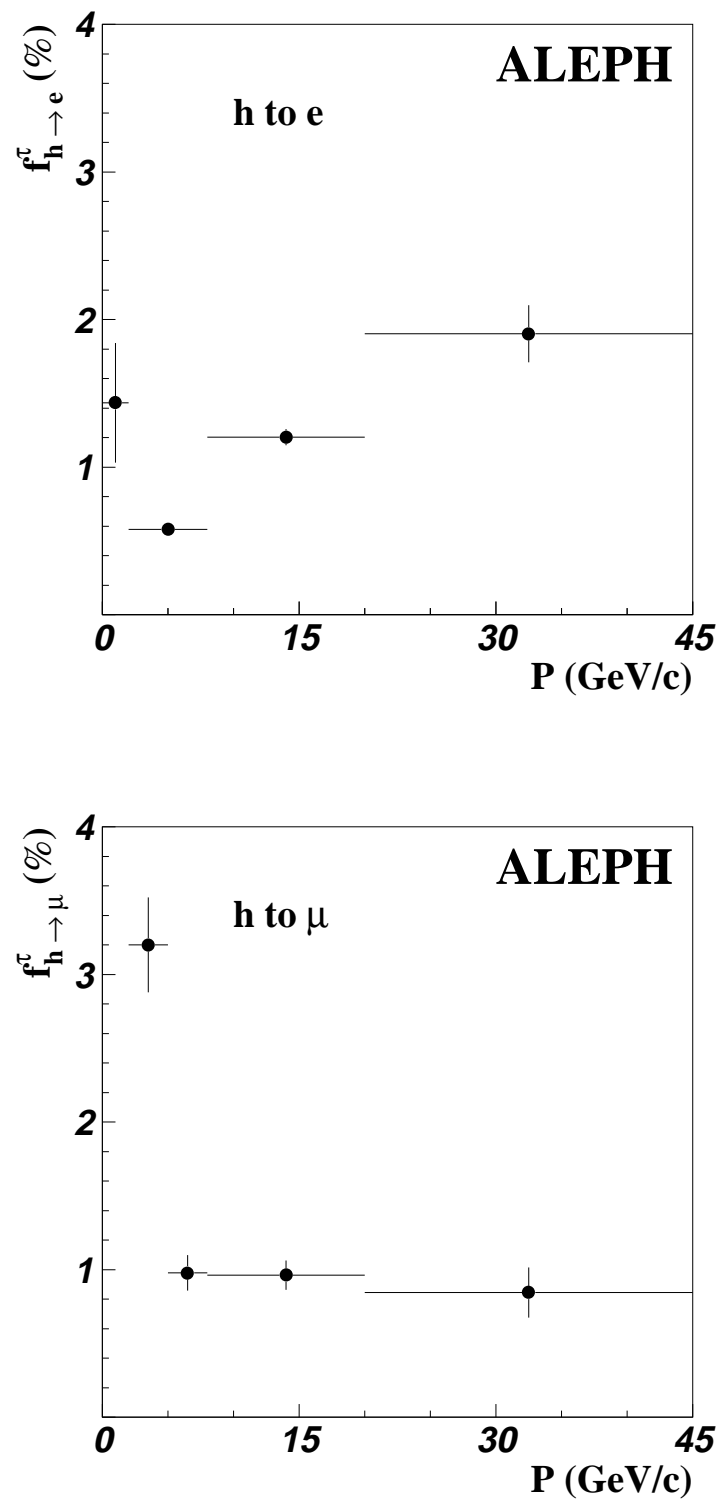

Figure 11: The measured hadron contamination in the final lepton samples for 1991-93 data as a function of momentum. 
estimates are given for less crucial, however important effects.

\subsection{Energy calibration and resolution}

Bhabha and $\mu \mu$ backgrounds are evaluated with the help of simulation for the shape of the distributions, but they are normalized using data through the procedure discussed in Section 4 . The energy distributions are sensitive to the detector resolution which could affect the background calculation if improperly understood. These effects are studied on samples of Bhabha and $\mu \mu$ events and compared to the respective Monte Carlo samples.

In the Monte Carlo Bhabha samples, the distribution of the jet energies is shifted by 1 $\mathrm{GeV}$ as compared to the data with some differences in the radiative tail. A detailed study of ECAL energy calibration and resolution performed for the different detector elements (endcaps and barrel) shows a consistent effect. Finally, a conservative range of $\pm 2 \mathrm{GeV}$ in the energy cut value yields a systematic uncertainty of $\pm 13 \%$ for the Bhabha contamination in the $\tau \tau$ sample and $\pm 7 \%$ in the electron sample.

A similar study is carried out for muons. Momentum calibration is quite good here as it is based only on tracking, but the resolution is underestimated in the simulation by $10 \%$. Changing the cut accordingly gives a systematic uncertainty of $\pm 14 \%$ and $\pm 16 \%$ for the $\mu \mu$ contamination in the $\tau \tau$ and muon samples, respectively.

The effect of calibration and resolution on $\tau \tau$ efficiency is quite small: $0.2 \%$ for $\tau \tau$ and $0.3 \%$ for the leptonic samples. In any case, it is already taken into account with the procedure described in Section 4.4 .

\subsection{Other uncertainties}

Hadron contamination in the lepton sample is proportional to the one-prong hadronic branching ratio $(\sim 50 \%)$ which is known to $\pm 1 \%$ absolute [21]. The effect on the leptonic branching ratios is limited to $\pm 0.4 \%$.

The efficiency of the energy cuts depends on the $\tau$ polarisation: leptons from left-handed $\tau^{-}$'s are affected more by the Bhabha and $\mu \mu$ cuts than right-handed $\tau^{-}$'s, while the opposite is true for the $\gamma \gamma$ cuts and the $P>2 \mathrm{GeV} / c$ condition for $\mu$ identification. The relative efficiency difference between the two helicities is estimated by Monte Carlo to be $2.4 \%$ for electrons and $5.8 \%$ for muons, because of the $2 \mathrm{GeV} / c$ cut applied to muons. An uncertainty of 0.02 on the $\tau$ polarisation [22] translates into a $0.2 \%(0.4 \%)$ uncertainty on the electron (muon) efficiency.

Finally, Table 5 summarizes the main uncertainties for the leptonic branching ratios. 


\begin{tabular}{|l|c|c|}
\hline & $e$ & $\mu$ \\
\hline statistics (data) & 6.5 & 6.4 \\
\hline statistics (Monte Carlo) & 1.3 & 1.3 \\
$\tau \tau$ selection & 1.5 & 1.5 \\
non- $\tau$ background & 1.6 & 1.6 \\
$l$ selection & 1.0 & 0.7 \\
single track & 0.7 & 0.4 \\
identification efficiency & 1.0 & 1.0 \\
hadron contamination & 0.7 & 0.8 \\
hadronic branching ratios & 0.4 & 0.4 \\
$\tau$ polarisation & 0.2 & 0.4 \\
\hline total systematics & 3.1 & 3.0 \\
\hline
\end{tabular}

Table 5: Summary of relative uncertainties for leptonic branching ratios (in $\%$ ).

\section{$7 \quad$ Results}

The statistics of the lepton samples is given in Table 6 with a summary of the relevant efficiencies, background fractions and their respective systematic uncertainties. The branching ratios are derived by means of Eq. 1 and the results for the different periods of data-taking are compared in Fig. 12.

\begin{tabular}{|l|c|c|}
\hline & $e$ & $\mu$ \\
\hline$N_{l}$ & 20571 & 20745 \\
$\varepsilon_{l}^{\text {sel }}$ & $0.7689 \pm 0.0013$ & $0.7923 \pm 0.0011$ \\
$\varepsilon_{l}^{I D}$ & $0.9292 \pm 0.0013$ & $0.9386 \pm 0.0012$ \\
$f_{l}^{\text {non- } \tau}$ & $0.0118 \pm 0.0014$ & $0.0090 \pm 0.0014$ \\
$f_{h \rightarrow l}^{\tau} \rightarrow$ & $0.0126 \pm 0.0007$ & $0.0110 \pm 0.0008$ \\
\hline
\end{tabular}

Table 6: Summary of the lepton samples.

The results for the different years are in fair agreement: the probabilities for all the measurements to have fluctuated around the central values more than expected from the quoted uncertainties is $10 \%$ for electrons and $25 \%$ for muons. Since both $B_{e}$ and $B_{\mu}$ show some similar behaviour between the most precise data sets of 1992 and 1993, much effort was devoted to find a possible common systematic effect. All the components entering in Eq. 1 were carefully and systematically examined. No significant difference is observed for $\varepsilon_{\tau \tau}^{\text {sel }}(<1.2 \%), \varepsilon_{l}^{\text {sel }}(<1.8 \%), f_{l}^{\text {non }} \tau(<1.7 \%), f_{h \rightarrow l}^{\tau}(<1.2 \%)$, and $\varepsilon_{l}^{I D}(<1.0 \%)$. More global checks were performed on the lepton momentum spectrum showing that the 1993/1992 "excess" is independent of momentum. Also the identification of the $\tau$ decay opposite to the 


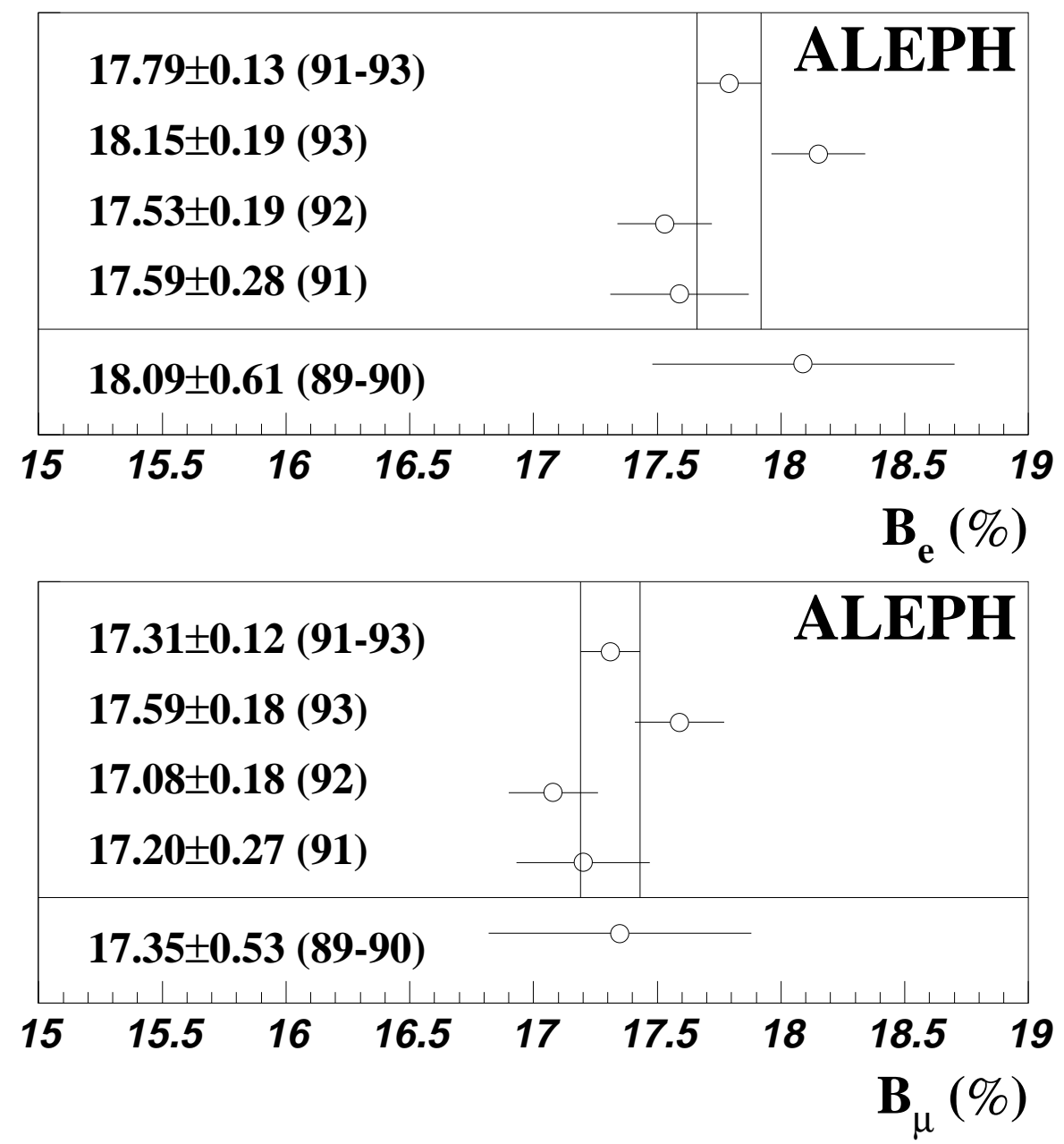

Figure 12: Measurements of the $\tau$ leptonic branching ratios for the different data sets. Results from 1989-90 data are already published [11]. 
lepton hemisphere revealed no significant correlation for purely leptonic final states. Finally, it was checked that off-peak data (taken in the 1993 energy scan where non- $\tau$ backgrounds are higher) were not responsible for larger branching ratios. In summary, no systematic effect is found beyond the quoted systematic uncertainties and the only remaining explanation is a statistical fluctuation in the number of electrons (mostly) and muons. This conclusion is strengthened by the results of the global analysis [21] where it is observed that the "excess" of leptons in the 1993 data compared to 1991-92 is not correlated to the drop of a specific hadron channel (say $\tau \rightarrow \nu_{\tau} \pi$ or $\tau \rightarrow \nu_{\tau} \rho$ ) as could be expected from a misidentification problem, but is rather uniformly balanced by all hadronic modes.

The only relevant observable in the leptonic channels is the lepton momentum. Fig. 13 and Fig. 14 show the respective track momentum distributions for electrons and muons. They are in good agreement with the simulation including backgrounds and with the Standard Model spectrum. The electron data distribution shows some excess near the end point of the spectrum above the Monte Carlo prediction. This cannot be explained by an underestimate of the Bhabha background since the calorimetric energy distributions for data and Monte Carlo are in excellent agreement in this region. Also, the particle content of the opposite hemispheres is completely consistent with the expectation from $\tau \tau$ events. Finally a visual scanning of the events revealed no unforeseen contamination. The effect is probably related to a tail in the momentum resolution function for electrons, as the muons do not show an excess at the same level. Since all checks performed provide no evidence for extra background, it is fair to assume that the apparent excess above $43 \mathrm{GeV} / c(1.9 \%$ of the electrons) should not affect the measurement of $B_{\epsilon}$.

The final results of this analysis are obtained by averaging over 1991-93 data:

$$
\begin{aligned}
& B_{e}=17.79 \pm 0.12(\text { stat }) \pm 0.06(\text { syst })(\%) \\
& B_{\mu}=17.31 \pm 0.11(\text { stat }) \pm 0.05(\text { syst })(\%)
\end{aligned}
$$

with a correlation coefficient of -0.11 for the total errors.

The overall precision achieved is $7 \%$ for both $B_{e}$ and $B_{\mu}$. These new measurements agree well with the published ALEPH values from 1989-90 data [11], but they are more precise by a factor of 4.3. Consequently, no significant improvement in the errors and no practical change in the final values are obtained if the published values are added to the new results. The above results are consistent within the uncertainties with those obtained in a global analysis of all $\tau$ branching fractions [21].

In Fig. 15, the ALEPH results are compared with those from other experiments. They agree well with the average values of other results, but they are significantly more precise: the 

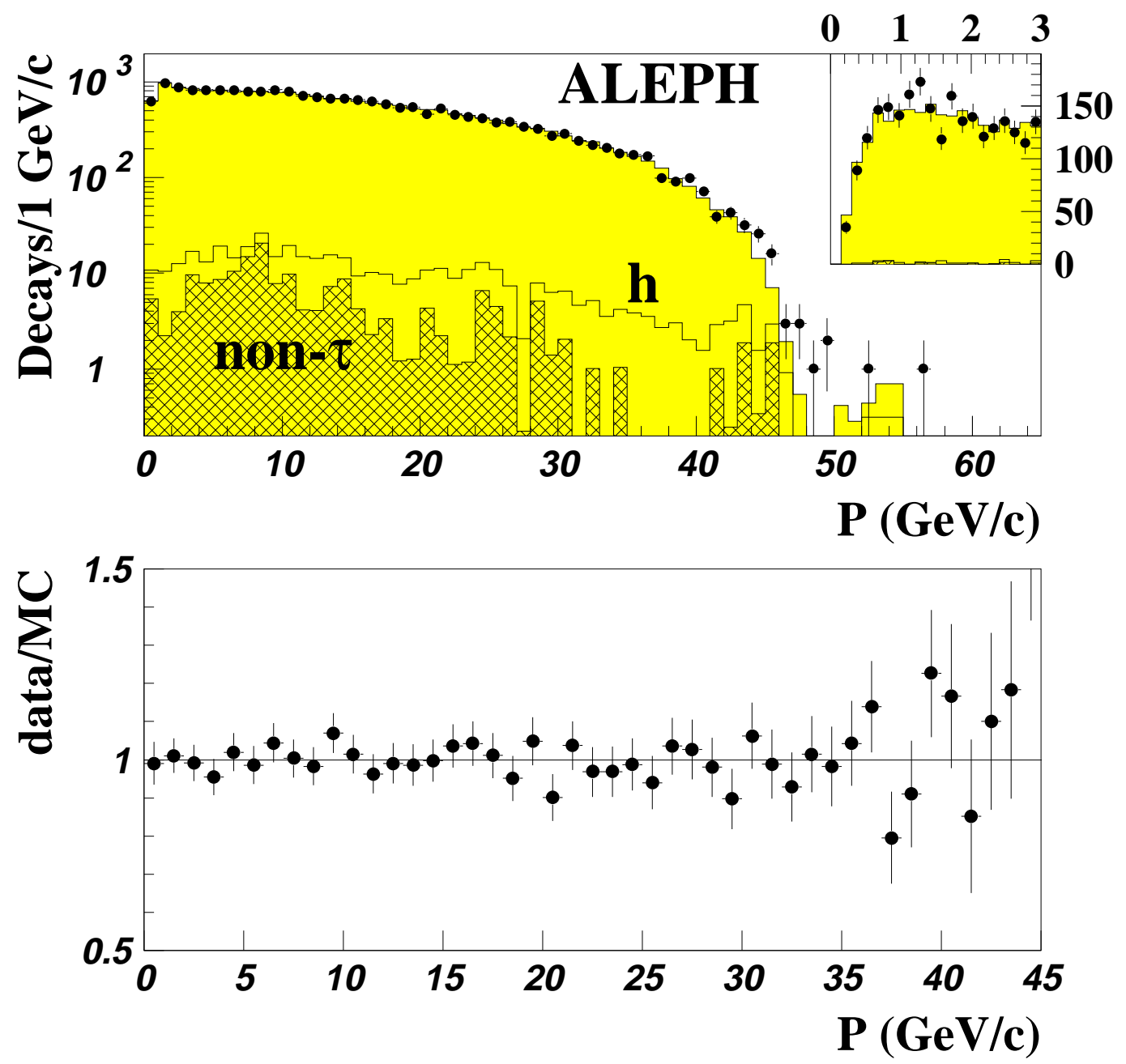

Figure 13: The electron momentum distributions (insert for low-momentum electrons in $0.15 \mathrm{GeV} / \mathrm{c}$ bins). The histogram is for the Monte Carlo with backgrounds indicated from hadron misidentification and non- $\tau$ sources. The two distributions are normalized to the same number of events. The lower plot shows the ratio of the distributions for the data and the Monte Carlo. 

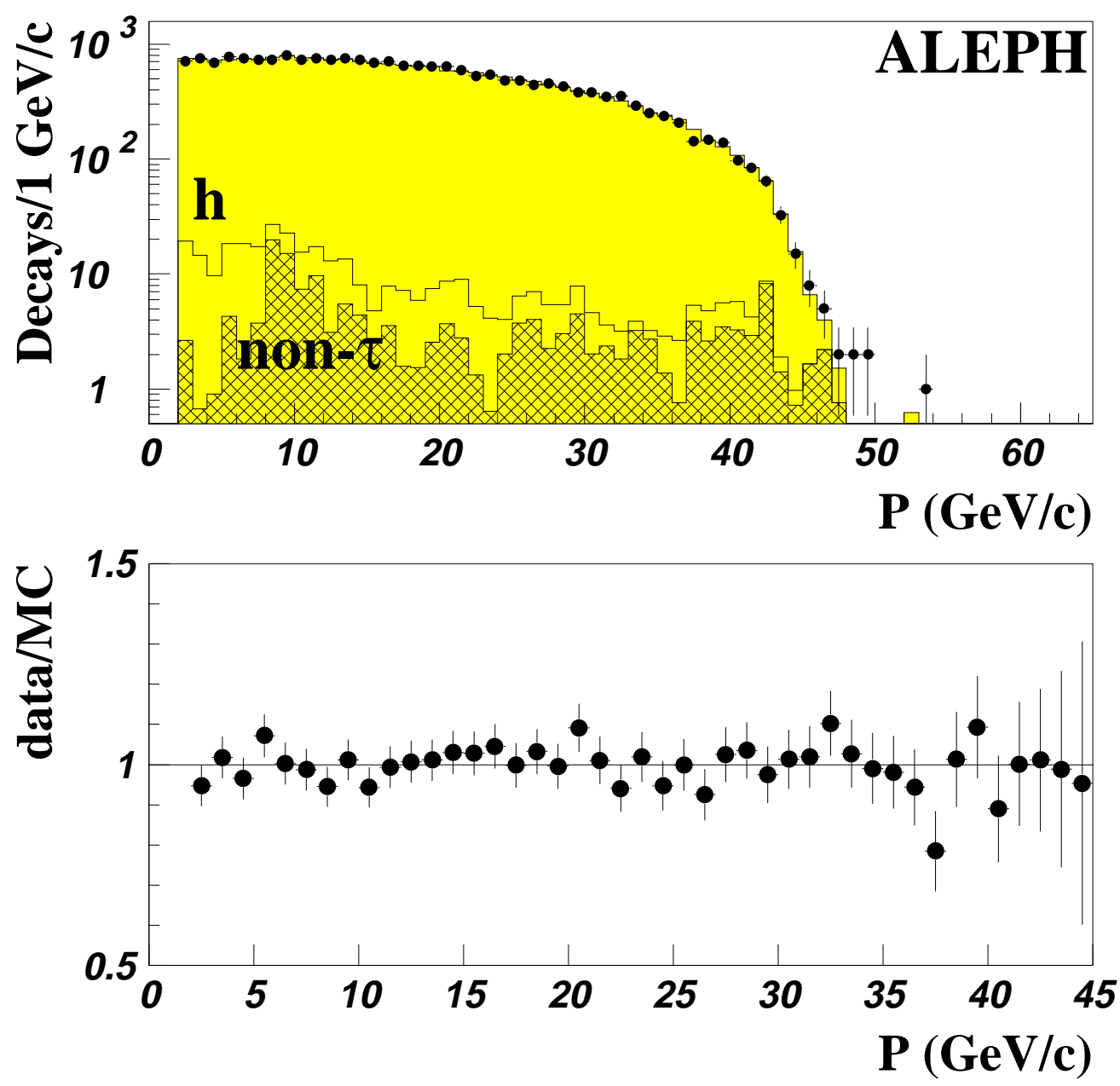

Figure 14: The muon momentum distributions. The histogram is for the Monte Carlo with backgrounds indicated from hadron misidentification and non- $\tau$ sources. The two distributions are normalized to the same number of events. The lower plot shows the ratio of the distributions for the data and the Monte Carlo. 
previous world averages [3] are $^{2} B_{e}=(17.90 \pm 0.17) \%$ and $B_{\mu}=(17.44 \pm 0.23) \%$, and the recent measurements of $B_{e}=(18.04 \pm 0.33) \%$ and $B_{\mu}=(17.36 \pm 0.27) \%$, and $B_{e}=(17.51 \pm 0.39) \%$ and $B_{\mu}=(17.02 \pm 0.31) \%$ are obtained by OPAL [24] and DELPHI [25], respectively.

\section{Tests of lepton universality in W couplings}

In the standard $\mathrm{V}-\mathrm{A}$ theory with leptonic coupling $g_{l}$ at the Wl $\bar{\nu}_{l}$ vertex, the $\tau$ leptonic partial width can be computed, including radiative corrections [2] and neglecting neutrino masses $^{3}$ :

$$
\Gamma\left(\tau \rightarrow \nu_{\tau} l \bar{\nu}_{l}(\gamma)\right)=\frac{G_{\tau} G_{l} m_{\tau}^{5}}{192 \pi^{3}} f\left(\frac{m_{l}^{2}}{m_{\tau}^{2}}\right) \delta_{W}^{\tau} \delta_{\gamma}^{\tau},
$$

where

$$
\begin{aligned}
G_{l} & =\frac{g_{l}^{2}}{4 \sqrt{2} M_{W}^{2}} \\
\delta_{W}^{\tau} & =1+\frac{3}{5} \frac{m_{\tau}^{2}}{M_{W}^{2}} \\
\delta_{\gamma}^{\tau} & =1+\frac{\alpha\left(m_{\tau}\right)}{2 \pi}\left(\frac{25}{4}-\pi^{2}\right) \\
f(x) & =1-8 x+8 x^{3}-x^{4}-12 x^{2} \ln x .
\end{aligned}
$$

Numerically, the $W$ propagator correction and the radiative corrections are small:

$$
\delta_{W}^{\tau}=1+2.9 \cdot 10^{-4} \quad \delta_{\gamma}^{\tau}=1-43.2 \cdot 10^{-4} .
$$

\subsection{Test of $\mu-e$ universality}

Taking the ratio of the two leptonic branching fractions, a direct test of $\mu-e$ universality is obtained

$$
\frac{B_{\mu}}{B_{e}}=\frac{f\left(\frac{m_{\mu}^{2}}{m_{\tau}^{2}}\right)}{f\left(\frac{m_{e}^{2}}{m_{\tau}^{2}}\right)}\left(\frac{g_{\mu}}{g_{e}}\right)^{2},
$$

where $f\left(\frac{m_{e}^{2}}{m_{\tau}^{2}}\right)$ is equal to one for all practical purposes. The results presented here yield

\footnotetext{
${ }^{2}$ The "average" values are quoted here rather than the "fit" values which are affected by all the hadronic modes. Problems are known to exist in the latter [23] introducing an undesirable bias in the leptonic modes.

${ }^{3} \mathrm{~A} \nu_{\tau}$ mass of $24 \mathrm{MeV} / c^{2}$, the present limit from ALEPH [36], would change the $\tau$ leptonic width by $1.5 \cdot 10^{-3}$, well below the experimental accuracy of the measurements discussed in this paper.
} 

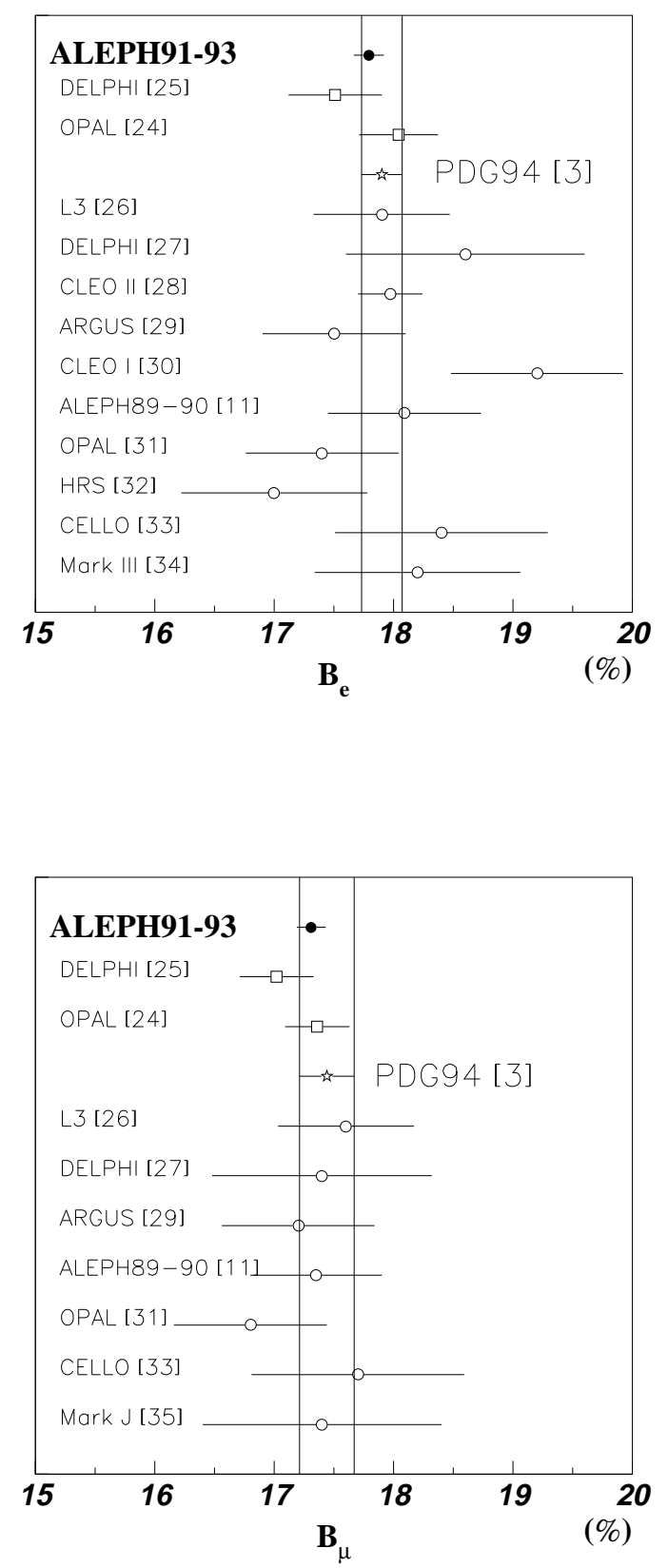

Figure 15: Comparison of the present results with published experiments with a total uncertainty less than 1\% absolute. PDG94 includes the results of experiments displayed below. 


$$
\frac{B_{\mu}}{B_{e}}=0.9732 \pm 0.0095(\text { stat }) \pm 0.0033(\text { syst }),
$$

where correlations in the statistical and systematical uncertainties have been taken into

account. Since $f\left(\frac{m_{\mu}^{2}}{m_{\tau}^{2}}\right)$ is computed to be 0.9726 , the above result is in excellent agreement with $\mu-e$ universality. Quantitatively, the result

$$
\frac{g_{\mu}}{g_{e}}=1.0002 \pm 0.0051
$$

is in agreement with the less precise values of $1.0009 \pm 0.0081$ obtained from the world-average leptonic branching ratios [3], and $0.994 \pm 0.012$ and $1.000 \pm 0.013$ from the recent OPAL [24] and DELPHI [25] analyses, respectively.

The result (11) is in agreement with the best test of $\mu-e$ universality of the $W$ couplings achieved in the comparison of the rates for $\pi \rightarrow \mu \bar{\nu}_{\mu}$ and $\pi \rightarrow e \bar{\nu}_{e}$ where the two most precise experiments [37] [38] can be combined [39] to yield $g_{\mu} / g_{e}=1.0012 \pm 0.0016$. Although the result from $\tau$ decay is less precise, it is nevertheless interesting as it checks the coupling to a transverse $W$ (helicity $= \pm 1$ ) while the $\pi$ decays measure the coupling to a longitudinal $W$ (helicity $=0$ ). It is conceivable that either approach could be sensitive to different non-standard corrections to universality and the two tests are therefore complementary.

Since $B_{e}$ and $B_{\mu}$ are consistent with $\mu-e$ universality their values can be combined, taking common errors into account, into a consistent leptonic branching ratio for a massless lepton

$$
B_{l}^{\left(m_{l}=0\right)}=17.793 \pm 0.071(\text { stat }) \pm 0.043(\text { syst })(\%) .
$$

\subsection{Tests of $\tau-\mu$ and $\tau-e$ universality}

Comparing the rates for $\tau \rightarrow \nu_{\tau} e \bar{\nu}_{e}(\gamma), \tau \rightarrow \nu_{\tau} \mu \bar{\nu}_{e}(\gamma)$ and $\mu \rightarrow \nu_{\mu} e \bar{\nu}_{e}(\gamma)$ provides direct checks of the universality of $\tau-\mu-e$ couplings. Taking the relevant ratios, one obtains

$$
\begin{aligned}
& \left(\frac{g_{\tau}}{g_{\mu}}\right)^{2}=\frac{\tau_{\mu}}{\tau_{\tau}}\left(\frac{m_{\mu}}{m_{\tau}}\right)^{5} B_{e} \frac{f\left(\frac{m_{e}^{2}}{m_{\mu}^{2}}\right)}{f\left(\frac{m_{e}^{2}}{m_{\tau}^{2}}\right)} \Delta_{W} \Delta_{\gamma} \\
& \left(\frac{g_{\tau}}{g_{e}}\right)^{2}=\frac{\tau_{\mu}}{\tau_{\tau}}\left(\frac{m_{\mu}}{m_{\tau}}\right)^{5} B_{\mu} \frac{f\left(\frac{m_{e}^{2}}{m_{\mu}^{2}}\right)}{f\left(\frac{m_{\mu}^{2}}{m_{\tau}^{2}}\right)} \Delta_{W} \Delta_{\gamma},
\end{aligned}
$$

where $f\left(\frac{m_{e}^{2}}{m_{\mu}^{2}}\right)=0.9998$ and

$$
\Delta_{W}=\frac{\delta_{W}^{\mu}}{\delta_{W}^{\tau}}=1-2.9 \cdot 10^{-4}
$$




$$
\Delta_{\gamma}=\frac{\delta_{\gamma}^{\mu}}{\delta_{\gamma}^{\tau}}=1+8.5 \cdot 10^{-5} .
$$

Using $m_{\tau}=(1776.96 \pm 0.26) \mathrm{MeV} / c^{2}$ measured by BES [40], the most recent determination by ALEPH of the $\tau$ lifetime [41]

$$
\tau_{\tau}=293.7 \pm 2.7(\text { stat }) \pm 1.6(\text { syst })(\mathrm{fs}),
$$

the branching ratios measured in this analysis, and values for the other quantities from the Particle Data Group [3], universality can be tested:

$$
\begin{aligned}
& \frac{g_{\tau}}{g_{\mu}}=0.9943 \pm 0.0037\left(B_{e}\right) \pm 0.0053\left(\tau_{\tau}\right) \pm 0.0004\left(m_{\tau}\right) \\
& \frac{g_{\tau}}{g_{e}}=0.9946 \pm 0.0035\left(B_{\mu}\right) \pm 0.0053\left(\tau_{\tau}\right) \pm 0.0004\left(m_{\tau}\right) .
\end{aligned}
$$

Thus universality for $\tau-\mu$ and $\tau-e$ is verified with an accuracy of $6.5 \%$.

Alternatively, if universality is assumed for the light leptons $(e, \mu)$, it is possible to investigate the behaviour of the heavy $\tau$ lepton with a more stringent test using the combined leptonic branching ratio (12):

$$
\frac{g_{\tau}}{g_{e, \mu}}=0.9945 \pm 0.0023\left(B_{l}\right) \pm 0.0053\left(\tau_{\tau}\right) \pm 0.0004\left(m_{\tau}\right),
$$

as shown in Fig. 16. The result (18) is consistent with universality and agrees with the value obtained from the world average $[3,4](0.9928 \pm 0.0065)$ and the recent determination by OPAL $[24](1.007 \pm 0.007)$. 


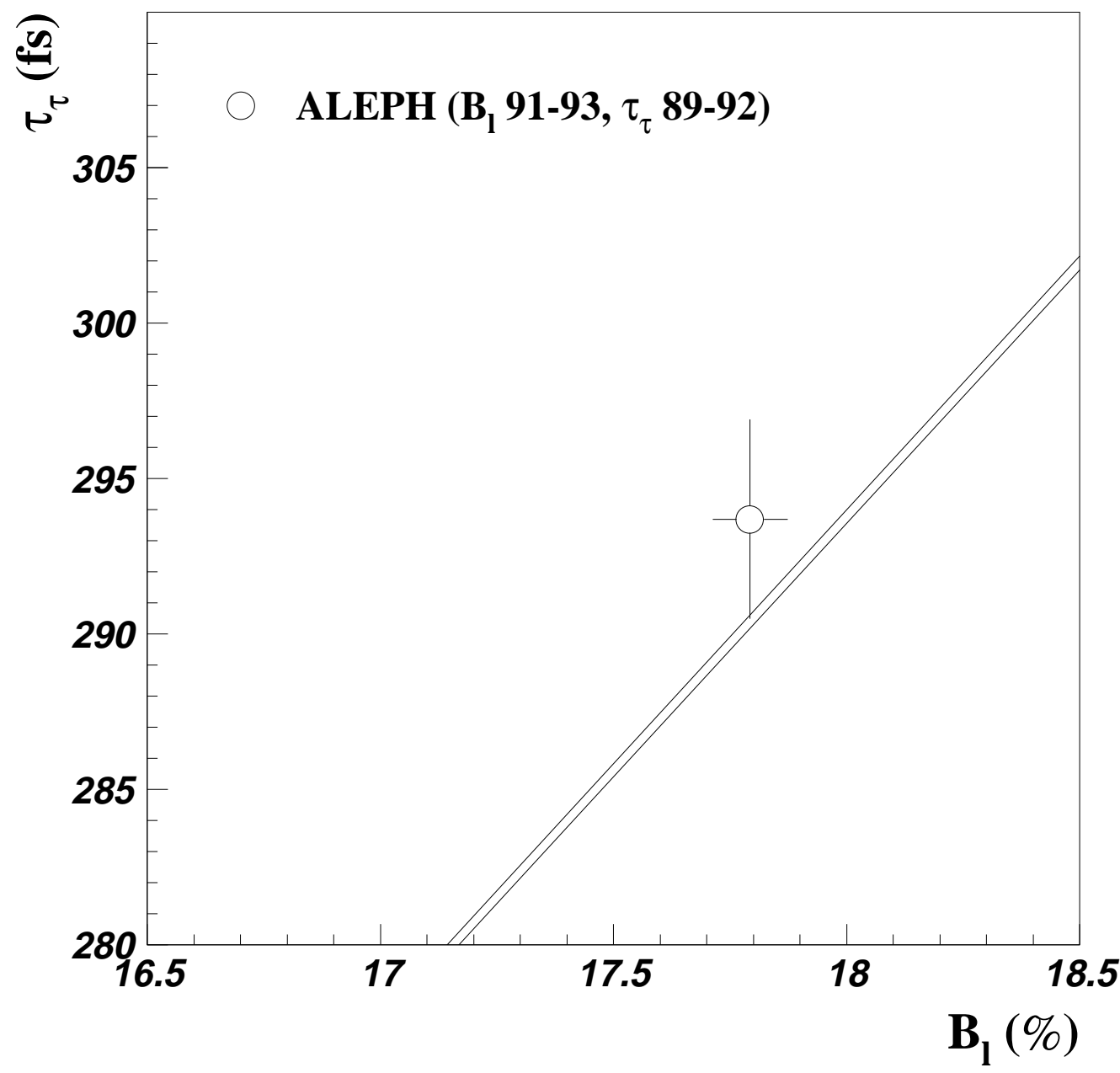

Figure 16: Test of $\tau$ universality with light leptons $(e, \mu)$ : the straight line is the prediction based on the assumption of universality with the uncertainty from the $\tau$ mass measurement [39] indicated. 


\section{Conclusions}

From a sample of $62249 \tau$-pair events selected from data taken with the ALEPH detector in 1991, 1992 and 1993, $20571 \tau \rightarrow \nu_{\tau} e \bar{\nu}_{e}(\gamma)$ and $20745 \tau \rightarrow \nu_{\tau} \mu \bar{\nu}_{\mu}(\gamma)$ decays are identified. The analysis is characterized by large efficiencies $(\sim 78 \%)$ and small background contamination $(\sim 1 \%)$. The results on the respective branching ratios are obtained:

$$
\begin{aligned}
& B_{e}=17.79 \pm 0.12(\text { stat }) \pm 0.06(\text { syst })(\%) \\
& B_{\mu}=17.31 \pm 0.11(\text { stat }) \pm 0.05(\text { syst })(\%) .
\end{aligned}
$$

Lepton universality in the charged weak current is observed to hold with a precision better than $1 \%$. From $B_{e}$ and $B_{\mu}, \mu-e$ universality is tested with

$$
\frac{g_{\mu}}{g_{e}}=1.0002 \pm 0.0051
$$

Combining the result on $B_{e}$ with the most recent determination of the $\tau$ lifetime by ALEPH yields a precise test of $\tau$ - $\mu$ universality

$$
\frac{g_{\tau}}{g_{\mu}}=0.9943 \pm 0.0065
$$

These two universality tests involving transverse $W$ couplings are the most precise to date obtained in a single experiment.

\section{Acknowledgements}

We wish to thank our colleagues from the accelerator divisions for the successful operation of LEP. We are indebted to the engineers and technicians in all our institutions for their contribution to the excellent performance of ALEPH. Those of us from non-member countries thank CERN for its hospitality. 


\section{References}

[1] D. Schaile, Proceedings of the XXVII International Conference on High Energy Physics (Glasgow, July 1994), to be published.

[2] W. Marciano and A. Sirlin, Phys. Rev. Lett. 61, (1988) 1815.

[3] Particle Data Group, L. Montanet et al., Phys. Rev. D50 (1994) 1173.

[4] M. Davier, Proceedings of the 3rd Workshop on Tau Lepton Physics, Montreux 1994, L. Rolandi ed., Nucl. Phys. (Proc. Suppl.) (1995).

[5] E. Braaten, S. Narison and A. Pich, Nucl. Phys. B373 (1992) 581.

[6] ALEPH Collaboration, D. Buskulic et al., Phys. Lett. B307 (1993) 209.

[7] L. Duflot (ALEPH Coll.), Proceedings of the 3rd Workshop on Tau Lepton Physics, Montreux 1994, L. Rolandi ed., Nucl. Phys. (Proc. Suppl.) (1995).

[8] ALEPH Collaboration, D. Buskulic et al., Phys. Lett. B346 (1995) 379.

[9] ALEPH Collaboration, D. Decamp et al., Nucl. Inst. and Meth. A294 (1990) 121.

[10] ALEPH Collaboration, D. Buskulic et al., Phys. Lett. B332 (1994) 209.

[11] ALEPH Collaboration, D. Decamp et al., Z. Phys. C54 (1992) 211.

[12] ALEPH Collaboration, D. Buskulic et al., Nucl. Inst. and Meth. A360 (1995) 481.

[13] S. Jadach, B.F.L. Ward, and Z. Wa̧s, Comp. Phys. Comm. 79 (1994) 503; S. Jadach et al., Comp. Phys. Comm. 76 (1993) 361.

[14] For details see H.J. Park, Ph.D thesis, Université de Paris-Sud, LAL 95-08, 1995.

[15] ALEPH Collaboration, D. Decamp et al., Z. Phys. C62 (1994) 539.

[16] T. Sjöstrand, Comp. Phys. Comm. 39 (1986) 347.

[17] ALEPH Collaboration, D. Buskulic et al., Z. Phys. C66 (1995) 3.

[18] M. Böhm, A. Denner and W. Hollik, Nucl. Phys. B304 (1988) 687; F.A. Berends, R. Kleiss and W. Hollik, Nucl. Phys. B304 (1988) 712.

[19] H. Anlauf et al., Comp. Phys. Comm. 79 (1994) 466.

[20] J.M. Hilgart, R. Kleiss and F. LeDiberder, Comp. Phys. Comm. 75 (1993) 191.

[21] ALEPH Collaboration, D. Buskulic et al., to be published. 
[22] ALEPH Collaboration, D. Buskulic et al., CERN-PPE/95-023, 1995. Submitted to Z. Phys..

[23] M. Davier, Proceedings of the 2nd Workshop on Tau Lepton Physics, Columbus 1992, K.K. Gan ed., World Scientific (1993).

[24] OPAL Collaboration, R. Akers et al., Z. Phys. C66 (1995) 543.

[25] DELPHI Collaboration, P. Abreu et al., CERN-PPE/95-114, 1995.

[26] L3 Collaboration, O. Adriani et al., Phys. Rep., 236 (1993) 1.

[27] DELPHI Collaboration, P. Abreu et al., Z. Phys., C55 (1992) 555.

[28] CLEO Collaboration, D.S. Akerib et al., Phys. Rev. Lett. 69 (1992) 3610; (erratum) 71 (1993) 3395.

[29] ARGUS Collaboration, H. Albrecht et al., Z. Phys., C53 (1992) 367.

[30] CLEO Collaboration, R. Ammar et al., Phys. Rev. D45 (1992) 3976.

[31] OPAL Collaboration, G. Alexander et al., Phys. Lett. B266 (1991) 201.

[32] HRS Collaboration, S. Abachi et al., Phys. Rev. D41 (1990) 1414.

[33] CELLO Collaboration, H.J. Behrend et al., Z. Phys., C46 (1990) 537.

[34] Mark III Collaboration, R.M. Baltrusaitis et al., Phys. Rev. Lett. 55 (1985) 1842.

[35] Mark J Collaboration, B. Adeva et al., Phys. Rev. D38 (1988) 2665.

[36] ALEPH Collaboration, D. Buskulic et al., Phys. Lett. B349 (1995) 585.

[37] D.I. Britton et al., Phys. Rev. Lett. 68 (1992) 3000.

[38] C. Czapek et al., Phys. Rev. Lett. 70 (1993) 17.

[39] W. Marciano, Proceedings of the 3rd Workshop on Tau Lepton Physics, Montreux 1994, L. Rolandi ed., Nucl. Phys. (Proc. Suppl.) (1995).

[40] Qi Nading (BES Coll.), Proceedings of the 3rd Workshop on Tau Lepton Physics, Montreux 1994, L. Rolandi ed., Nucl. Phys. (Proc. Suppl.) (1995).

[41] ALEPH Collaboration, D. Buskulic et al., CERN-PPE/95-128, 1995. 